\title{
Synchronization of two non-scalar-coupled limit-cycle oscillators
}

\author{
M.V. Ivanchenko ${ }^{\mathrm{a}, *}$, G.V. Osipov ${ }^{\mathrm{a}}$, V.D. Shalfeev ${ }^{\mathrm{a}}$, J. Kurths $^{\mathrm{b}}$ \\ a Department of Radiophysics, Nizhny Novgorod University, 23 Gagarin Avenue, 603600 Nizhny Novgorod, Russia \\ ${ }^{\mathrm{b}}$ Institute of Physics, University of Potsdam, 10 Am Neuen Palais, D-14415 Potsdam, Germany
}

Received 2 July 2003; accepted 25 September 2003

Communicated by Y. Kuramoto

\begin{abstract}
Being one of the fundamental phenomena in nonlinear science, synchronization of oscillations has permanently remained an object of intensive research. Development of many asymptotic methods and numerical simulations has allowed an understanding and explanation of various phenomena of self-synchronization. But even in the classical case of coupled van der Pol oscillators a full description of all possible dynamical regimes, their mutual transitions and characteristics is still lacking. We present here a study of the phenomenon of mutual synchronization for two non-scalar-coupled non-identical limit-cycle oscillators and analyze phase, frequency and amplitude characteristics of synchronization regimes. A series of bifurcation diagrams that we obtain exhibit various regions of qualitatively different behavior. Among them we find mono-, bi- and multistability regions, beating and "oscillation death" ones; also a region, where one of the oscillators dominates the other one is observed. The frequency characteristics that we obtain reveal three qualitatively different types of synchronization: (i) on the mean frequency (the in-phase synchronization), (ii) with a shift from the mean frequency caused by a conservative coupling term (the anti-phase synchronization), and (iii) on the frequency of one of the oscillators (when one oscillator dominates the other).
\end{abstract}

(C) 2003 Elsevier B.V. All rights reserved.

PACS: 05.45.Xt

Keywords: Synchronization; Coupled oscillators; Bifurcations

\section{Introduction}

Since the first observation of synchronization made by Huygens [1] in the 17th century this phenomenon has attracted much attention of researchers (for a review, see Refs. [2,3]). It was mainly caused by the fact that this phenomenon is characteristic of many processes observed in nature and science. Appleton [4] and van der Pol [5] have shown the possibility of synchronization of triode generator by a weak external periodic signal. External synchronization of self-oscillatory systems was studied by Andronov and Vitt [6,7] and Mandelshtam and Papaleksi [8]. Mutual synchronization of two quasi-harmonic self-oscillators was first studied by Mayer [9] and Gaponov [10]. For self-oscillators in relaxation regime externally forced synchronization was investigated

* Corresponding author. Tel.: +83-126-56242; fax: +83-126-56416.

E-mail address: ivanchenko@mail.nnov.ru (M.V. Ivanchenko). 
by Cartwright and Littlewood [11,12]; mutual synchronization by Bremsen and Feinberg [13] and Teodorchik [14].

More recent studies show permanent interest in the problem of mutual synchronization of limit-cycle oscillators. The limits of strong and small (compared to damping in the isolated oscillator) coupling were studied in Refs. [15,16]. The appearance of the "oscillator death" effect in ensembles of globally coupled oscillators with randomly distributed frequencies was investigated in Ref. [17]. In Refs. [18,19] synchronization of (isochronous and non-isochronous as well) limit-cycle oscillators (in case of coupling being of the same order as damping in the isolated oscillator) was quite scrupulously studied for some types of coupling. Synchronization phenomena in ensembles of coupled limit-cycle oscillators have also been intensively studied in recent years, see Refs. [20-22]. In Ref. [23] relevant results were obtained for the complex Ginzburg-Landau equation with spatial non-uniformity of natural frequency. Nevertheless, new dynamical regimes are still being found even in the classical case of two coupled van der Pol oscillators.

In the present paper synchronization between two coupled non-identical limit-cycle oscillators will be studied. We treat a quite general type of coupling that combines dissipative and conservative couplings. The mismatches between the interacting systems will also be of different nature (in the frequency and the amplitude ones). The existence of synchronization regimes and their phase, frequency and amplitude characteristics will be studied in dependence upon these parameters. The mono-, bi- and multistability properties of synchronization between such systems will be investigated in detail and illustrated by bifurcation diagrams in the parameter space. An interval of optimum coupling parameters, for which synchronization persists for arbitrary large mismatches between the systems, is found, and an explanation of this phenomenon will be presented.

This paper is organized as follows. In Section 2 we describe the model under study. In Section 3 we shortly discuss previously obtained results, which include a quite detailed description of well-studied cases of purely dissipative and purely conservative types of coupling. In Section 4 we present numerical results for the case when both types of coupling are introduced and only the frequency mismatch exists. The properties of correspondent synchronization regimes will be discussed in Section 5. In Section 6 the combination of frequency and amplitude mismatches is considered and properties of synchronization are studied. In Section 7 we summarize our results.

\section{Mathematical model}

In our work we study a system of two non-scalar-coupled non-identical van der Pol oscillators, which, when isolated, differ in frequency and amplitude of stationary regime. In general, the coupling is supposed to contain both dissipative and conservative terms. This system is described by the following equations:

$$
\begin{aligned}
& \ddot{x}_{1}+x_{1}=\mu\left(1-x_{1}^{2}\right) \dot{x}_{1}+\mu \alpha\left(x_{2}-x_{1}\right)+\mu \beta\left(\dot{x}_{2}-\dot{x}_{1}\right), \\
& \ddot{x}_{2}+(1+\mu \Delta) x_{2}=\mu\left(1+\gamma-x_{2}^{2}\right) \dot{x}_{2}+\mu \alpha\left(x_{1}-x_{2}\right)+\mu \beta\left(\dot{x}_{1}-\dot{x}_{2}\right),
\end{aligned}
$$

where we assume $\mu \ll 1, \alpha$ and $\beta$ regulate the rate of the conservative and dissipative coupling, respectively, $\gamma$ and $\Delta$ define the amplitude and the frequency mismatches. We would like to stress that, in spite of the coupling's being of quite general form, it is, at the same time, characteristic of van der Pol oscillators and easy to be organized. By averaging (1) we obtain

$$
\dot{z}_{1}=z_{1}\left(1-z_{1} \bar{z}_{1}\right)+(\beta-\mathrm{i} \alpha)\left(z_{2}-z_{1}\right), \quad \dot{z}_{2}=z_{2}\left(1+\gamma-z_{2} \bar{z}_{2}\right)+\mathrm{i} \Delta z_{2}+(\beta-\mathrm{i} \alpha)\left(z_{1}-z_{2}\right),
$$


$z_{1}$ and $z_{2}$ being complex amplitudes. Conversion to real amplitudes and phases $z_{1}=\left(R_{1} / 2\right) \mathrm{e}^{-\mathrm{i} \phi_{1}}, z_{2}=\left(R_{2} / 2\right) \mathrm{e}^{-\mathrm{i} \phi_{2}}$ yields

$$
\begin{aligned}
& \dot{R}_{1}=R_{1}\left(1-\beta-\frac{1}{4} R_{1}^{2}\right)+R_{2}\left(\alpha \sin \left(\phi_{1}-\phi_{2}\right)+\beta \cos \left(\phi_{1}-\phi_{2}\right)\right), \\
& \dot{R}_{2}=R_{2}\left(1+\gamma-\beta-\frac{1}{4} R_{2}^{2}\right)+R_{1}\left(-\alpha \sin \left(\phi_{1}-\phi_{2}\right)+\beta \cos \left(\phi_{1}-\phi_{2}\right)\right), \\
& R_{1} \dot{\phi}_{1}=-\alpha R_{1}+R_{2}\left(\alpha \cos \left(\phi_{1}-\phi_{2}\right)-\beta \sin \left(\phi_{1}-\phi_{2}\right)\right), \\
& R_{2} \dot{\phi}_{2}=-(\alpha+\Delta) R_{2}+R_{1}\left(\alpha \cos \left(\phi_{1}-\phi_{2}\right)+\beta \sin \left(\phi_{1}-\phi_{2}\right)\right) .
\end{aligned}
$$

Writing this system in terms of the phase difference $\phi=\phi_{1}-\phi_{2}$, we get the system that we are going to deal with in order to study synchronization processes in the original system (1):

$$
\begin{aligned}
& \dot{R}_{1}=R_{1}\left(1-\beta-\frac{1}{4} R_{1}^{2}\right)+R_{2}(\alpha \sin \phi+\beta \cos \phi), \quad \dot{R}_{2}=R_{2}\left(1+\gamma-\beta-\frac{1}{4} R_{2}^{2}\right)+R_{1}(-\alpha \sin \phi+\beta \cos \phi), \\
& \dot{\phi}=\Delta+\alpha\left(\frac{R_{2}}{R_{1}}-\frac{R_{1}}{R_{2}}\right) \cos \phi-\beta\left(\frac{R_{2}}{R_{1}}+\frac{R_{1}}{R_{2}}\right) \sin \phi .
\end{aligned}
$$

As long as non-zero rest states of (4), which are solutions of

$$
\begin{aligned}
& \bar{R}_{1}\left(1-\beta-\frac{1}{4} \bar{R}_{1}^{2}\right)+\bar{R}_{2}(\alpha \sin \bar{\phi}+\beta \cos \bar{\phi})=0, \quad \bar{R}_{2}\left(1+\gamma-\beta-\frac{1}{4} \bar{R}_{2}^{2}\right)+\bar{R}_{1}(-\alpha \sin \bar{\phi}+\beta \cos \bar{\phi})=0 \\
& \Delta+\alpha\left(\frac{\bar{R}_{2}}{\bar{R}_{1}}-\frac{\bar{R}_{1}}{\bar{R}_{2}}\right) \cos \bar{\phi}-\beta\left(\frac{\bar{R}_{2}}{\bar{R}_{1}}+\frac{\bar{R}_{1}}{\bar{R}_{2}}\right) \sin \bar{\phi}=0
\end{aligned}
$$

correspond to synchronization regimes of (1) we focus our attention on analyzing these rest states of (4). Finding bifurcation curves, where the rest states change their stability, emerge or disappear allows us to obtain regions of qualitatively different behavior in the $(\alpha, \beta, \gamma, \Delta)$ parameter space. Besides, we get the boundaries, where limit cycles of (4) that correspond to quasi-periodic solutions of (1) (i.e. beating regimes) appear, and investigate the stability of the zero rest state (which is evidently not captured by (4) and (5)) performing linear analysis in (2).

\section{Previous results and general description}

Various special cases of (1) have been analyzed by many authors. Here we state their results briefly as we are going to address them later.

Purely dissipative coupling. A complete analysis of (1) in case of a purely dissipative coupling $(\alpha=0)$ and zero amplitude mismatch $(\gamma=0)$ is reported in Ref. [18]. In this case the following symmetry property takes place: (4) is invariant under the transformation $\bar{R}_{1} \rightarrow \bar{R}_{2}, \bar{R}_{2} \rightarrow \bar{R}_{1}$. The bifurcation diagram in the $(\beta, \Delta)$ plane is presented in Fig. 1.

Throughout the present paper the following symbols are used: $\mathrm{D}_{j}^{i}$ denotes the region in which $j$ is the total number of the rest states (including zero rest state), and $i$ the number of the stable ones. So $\mathrm{D}_{3}^{0}$ corresponds to the region where no stable steady state exists, i.e. the beating regime takes place. In $\mathrm{D}_{1}^{1}$ the only stationary (and stable) solution of (1) is the zero rest state $\left(x_{1,2}=\dot{x}_{1,2}=0\right)$, and the oscillator death takes place. In every other region the only stable solution possesses the constant phase difference $\bar{\phi}$. Further we are going to classify a solution as the in-phase one if $|\bar{\phi}| \leq \pi / 2$ and otherwise as the anti-phase one (it is justified by the fact that almost in the whole region of their existence these solutions do satisfy $|\bar{\phi}| \ll \pi / 2$ or $|\bar{\phi}-\pi| \ll \pi / 2$ ).

These designations allow to avoid an extensive and tedious description of bifurcations that occur in transition from one region to another one. Nevertheless, we would like to go over the details in interesting special cases, which were studied before. This also allows us to clear up our designation scheme. 


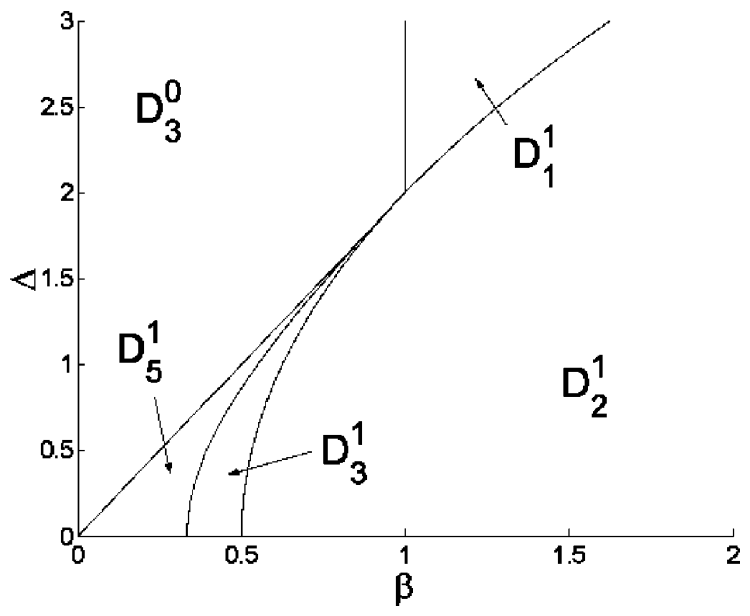

Fig. 1. Bifurcation diagram for $\alpha=0, \gamma=0$. In $D_{5}^{1}, D_{3}^{1}, D_{2}^{1}$ the in-phase synchronization exists. $D_{3}^{0}$ denotes the beating regime and $D_{1}^{1}$ corresponds to the "oscillator death" regime.

In the $(\beta, \Delta)$ parameter plane (Fig. 1) the following bifurcations are observed:

- If we move from $\mathrm{D}_{5}^{1}$ to $\mathrm{D}_{3}^{0}$, a stable and a saddle rest states of (4) merge and disappear through a saddle-node bifurcation. In general, if we cross the line between regions $\mathrm{D}_{j_{1}}^{i_{1}}$ and $\mathrm{D}_{j_{2}}^{i_{2}}$ and the number of stable rest states $i_{1,2}$ changes on $1\left(i_{2}=i_{1} \mp 1\right)$ and the total number of rest states $j_{1,2}$ changes on $2\left(j_{2}=j_{1} \mp 2\right)$, then a saddle-node bifurcation takes place. Nevertheless, there is a notable exception in case $\Delta=0$, which we are going to discuss a bit later.

- If we move from $D_{5}^{1}$ to $D_{3}^{1}$, then two saddle rest states of (4) (one with $\operatorname{dim} W^{\mathrm{s}}=1, \operatorname{dim} W^{\mathrm{u}}=2$ and another with $\left.\operatorname{dim} W^{\mathrm{s}}=2, \operatorname{dim} W^{\mathrm{u}}=1\right)$ merge and disappear through a saddle-saddle bifurcation. In general, if we move from region $\mathrm{D}_{j_{1}}^{i_{1}}$ to $\mathrm{D}_{j_{2}}^{i_{2}}$ and the number of stable rest states $i_{1,2}$ does not change $\left(i_{2}=i_{1}\right)$ and the total number of rest states $j_{1,2}$ changes on $2\left(j_{2}=j_{1} \mp 2\right)$, then a saddle-saddle bifurcation occurs.

- If we move from $D_{2}^{1}$ to $D_{1}^{1}$ (or from $D_{3}^{1}$ to $D_{2}^{1}$ ) then a stable (or saddle) rest state of (4) merges with the origin, which is always a solution of (1) and (2) (in (1) a stable (or saddle) limit cycle merges with the origin in a supercritical Andronov-Hopf bifurcation).

- At last, if we move from $\mathrm{D}_{3}^{0}$ to $\mathrm{D}_{1}^{1}$ two saddle limit cycles and a stable torus merge simultaneously with the origin in system (1), and the last one becomes stable. This bifurcation takes place only due to the symmetry property of the system, mentioned above, and does not exist if at least $\alpha \neq 0$ or $\gamma \neq 0$.

To conclude the treatment of this case, we underline that one synchronization regime exists in the regions $\mathrm{D}_{5}^{1}, \mathrm{D}_{3}^{1}$ and $\mathrm{D}_{2}^{1}$ and it is an in-phase one.

Purely conservative coupling. The results reported in Ref. [24] refer to the case of purely conservative coupling $(\beta=0)$ and zero amplitude mismatch $(\gamma=0)$. The bifurcation diagram in the parameter plane $(\alpha, \Delta)$ is presented in Fig. 2. In this case (4) is invariant under the transformation $\bar{R}_{1} \rightarrow \bar{R}_{2}, \bar{R}_{2} \rightarrow \bar{R}_{1}, \bar{\phi} \rightarrow \bar{\phi}-\pi$ that gives pairs of symmetric solutions.

The following bifurcations are observed:

- At the transition from $D_{7}^{2}$ to $D_{3}^{2}$ two saddle-saddle bifurcations occur simultaneously. In general, if we move from region $\mathrm{D}_{j_{1}}^{i_{1}}$ to $\mathrm{D}_{j_{2}}^{i_{2}}$ and the number of stable rest states $i_{1,2}$ does not change $\left(i_{2}=i_{1}\right)$ and the total number of rest states $j_{1,2}$ changes on $4\left(j_{2}=j_{1} \mp 4\right)$, then two saddle-saddle bifurcations occur simultaneously. 


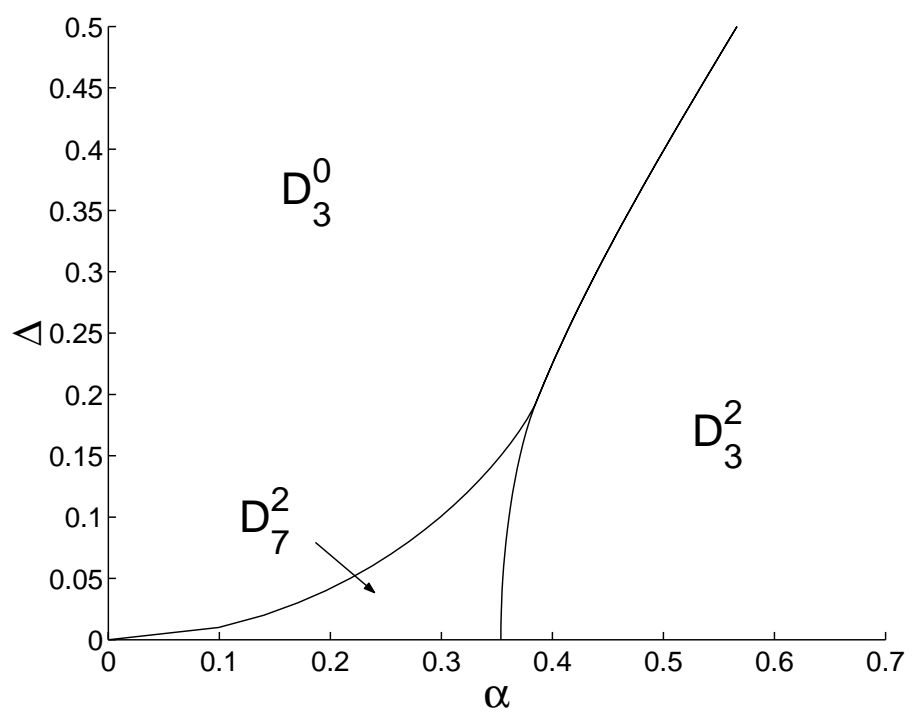

Fig. 2. Bifurcation diagram for $\beta=0$ and $\gamma=0$. Here $D_{7}^{2}$ and $D_{3}^{2}$ are regions of bistability. $\mathrm{D}_{3}^{0}$ corresponds to the beating regime.

- At the transition from $\mathrm{D}_{7}^{2}$ to $\mathrm{D}_{3}^{0}$ two saddle-node bifurcations occur simultaneously. In general, if we move from region $\mathrm{D}_{j_{1}}^{i_{1}}$ to $\mathrm{D}_{j_{2}}^{i_{2}}$ and the number of stable rest states $i_{1,2}$ changes on $2\left(i_{2}=i_{1} \mp 2\right)$ and the total number of rest states $j_{1,2}$ changes on $4\left(j_{2}=j_{1} \mp 4\right)$, then two saddle-node bifurcations occur simultaneously.

- At the transition from $\mathrm{D}_{3}^{2}$ to $\mathrm{D}_{3}^{0}$ two supercritical Andronov-Hopf bifurcations occur simultaneously. In general, if we move from region $\mathrm{D}_{j_{1}}^{i_{1}}$ to $\mathrm{D}_{j_{2}}^{i_{2}}$ and the number of stable rest states $i_{1,2}$ changes on $2\left(i_{2}=i_{1} \mp 2\right)$ and the total number of rest states $j_{1,2}$ does not change $\left(j_{2}=j_{1}\right)$, then two supercritical Andronov-Hopf bifurcations occur simultaneously.

In this case we observe bistability of synchronization regimes in the regions $\mathrm{D}_{3}^{2}$ and $\mathrm{D}_{7}^{2}$.

To complete the list of bifurcation types, which take place in our system, we should add the following ones, which do not occur in the cases described above:

- If by crossing a bifurcation curve between the regions $\mathrm{D}_{j_{1}}^{i_{1}}$ and $\mathrm{D}_{j_{2}}^{i_{2}}$ the number of stable rest states $i_{1,2}$ changes on $1\left(i_{2}=i_{1}-1\right)$ and the total number of rest states $j_{1,2}$ does not change $\left(j_{2}=j_{1}\right)$, then a stable focus of (4) becomes unstable through a supercritical Andronov-Hopf bifurcation and a stable limit cycle appears (e.g., the transition $\mathrm{D}_{3}^{2} \rightarrow \mathrm{D}_{3}^{1}$ (Figs. 3, 4, 6 and 12)).

- In the case $\Delta=0, j_{2}=j_{1} \mp 2$ and $i_{2}=i_{1} \mp 1$ corresponds to pitchfork bifurcation in system (4). Consequently, in system (1) $\mathrm{D}_{3}^{2} \rightarrow \mathrm{D}_{5}^{3}$ and $\mathrm{D}_{5}^{1} \rightarrow \mathrm{D}_{7}^{2}$ (Figs. 5 and 6) correspond to symmetry breaking bifurcations in which stable and two saddle limit cycles are involved.

Following this scheme one can easily identify which bifurcation each curve corresponds to.

In the case $\gamma=0, \alpha, \beta \ll 1$ an analytical approach has proved to be successful [16]. As the rest states near the unperturbed $\left(\alpha=\beta=0\right.$ ) values of amplitudes $\bar{R}_{1}^{0}=\bar{R}_{2}^{0}=2$ are sought, let

$$
\bar{R}_{1}=2+r_{1}, \quad \bar{R}_{2}=2+r_{2},
$$



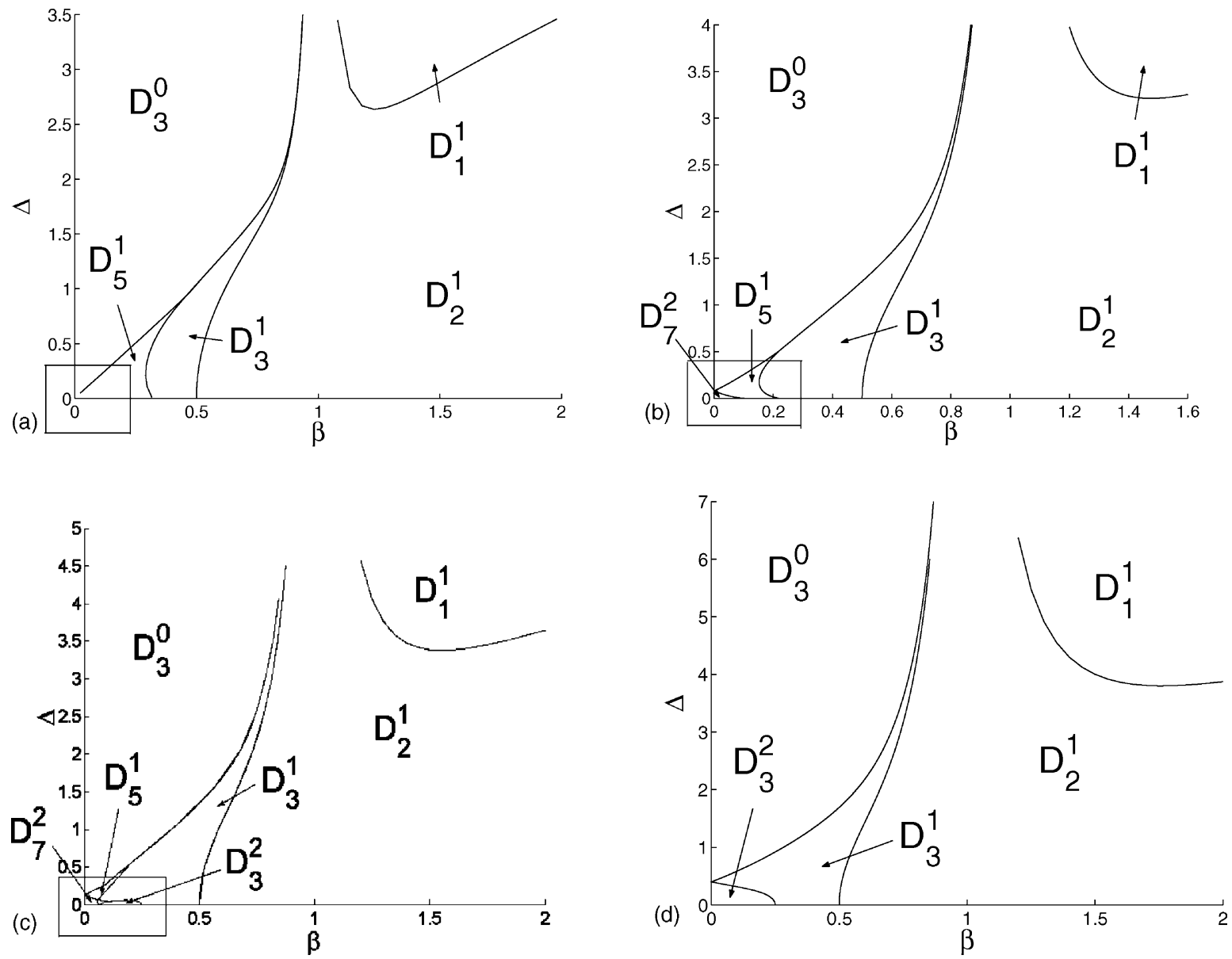

Fig. 3. (a) Bifurcation diagram for $\alpha=0.1, \gamma=0$; (b) bifurcation diagram for $\alpha=0.27, \gamma=0$; (c) bifurcation diagram for $\alpha=0.33$, $\gamma=0$; (d) bifurcation diagram for $\alpha=0.5, \gamma=0$ (regions framed with rectangle are presented enlarged in Fig. 4(a), (c) and (d), respectively). In the regions with the upper index " 2 " bistability takes place. Except for the case $\mathrm{D}_{1}^{1}$ (the "oscillator death"), the upper index " 1 " means monostable synchronization. As before, $\mathrm{D}_{3}^{0}$ corresponds to the beating regime.

where $r_{1}, r_{2} \ll 2$. Then

$$
r_{1}=\alpha \sin \bar{\phi}+\beta(\cos \bar{\phi}-1), \quad r_{2}=-\alpha \sin \bar{\phi}+\beta(\cos \bar{\phi}-1),
$$

gives the reduction of (4) to $\mathrm{O}\left(\alpha^{2}, \alpha \beta, \beta^{2}\right)$ :

$$
\Delta-\alpha^{2} \sin 2 \bar{\phi}-2 \beta \sin \bar{\phi}=0 .
$$

According to Ref. [16], (8) can have four, two, or no solutions at all. These solutions correspond to the rest states of (4), and among them one or two may be stable.

According to Ref. [16], the region of bistability of the in-phase $(\bar{\phi} \approx 0)$ and the anti-phase $(\bar{\phi} \approx \pi)$ solutions appears near $(0,0)$ in the $(\beta, \Delta)$ parameter plane (Figs. 3(a) and 4(a)), when $\alpha$ is slightly increasing from zero.

Besides, an analytical approach Ref. [18] allows to show the appearance of a bistability regime near $\beta=1 / 4$ when $\alpha=1 / 4$ and its further expansion down to $\beta=1 / 6$ (which gives the maximum of the bifurcation curve $\alpha^{2}=\beta-3 \beta^{2}$ shown in Fig. 4(b)) as $\alpha$ is increased (Fig. 4(b)) in case $\Delta=0, \gamma=0$ (the symmetry provides exact 

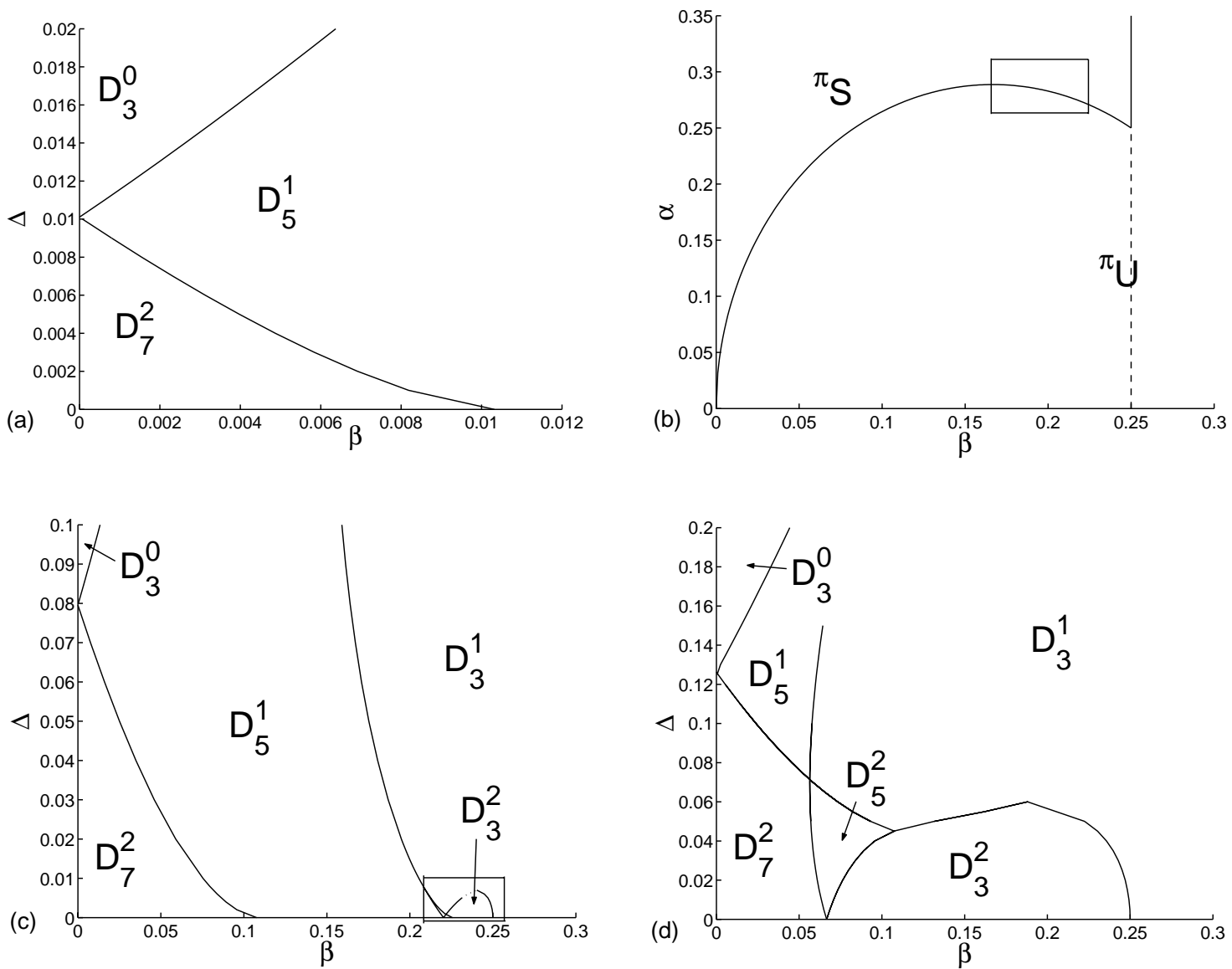

Fig. 4. (a) Part of the bifurcation diagram for $\alpha=0.1, \gamma=0$; (b) bifurcation curves for $\Delta=0, \gamma=0$ (barred region is presented enlarged in Fig. 5), in ${ }^{\pi} \mathrm{S}$ the exact anti-phase rest state $(\bar{\phi}=\pi)$ is stable, in ${ }^{\pi} \mathrm{U}$ it is unstable; a curve with quadratic form corresponds to the pitchfork bifurcation, the line $\beta=1 / 4$ denotes the Andronov-Hopf bifurcation; (c) part of the bifurcation diagram for $\alpha=0.27, \gamma=0$ (barred region is presented enlarged in Fig. 6); (d) part of the bifurcation diagram for $\alpha=0.33, \gamma=0$. In the regions with the upper index "2" bistability takes place. The upper index " 1 " means monostable synchronization. As before, $\mathrm{D}_{3}^{0}$ corresponds to the beating regime.

in- and anti-phase solutions ( $\bar{\phi}=0$ and $\bar{\phi}=\pi)$ ) that agree with Ref. [24] for $\beta=0, \Delta=0$ and $\gamma=0$. By crossing the curve $\alpha^{2}=\beta-3 \beta^{2}$, the anti-phase solution becomes involved in the pitchfork bifurcation; if $\alpha^{2}>\beta-3 \beta^{2}$. Then by crossing $\beta=1 / 4$, a supercritical Andronov-Hopf bifurcation occurs.

In Ref. [19] the equations of a form similar to (1) were analyzed. However, the assumptions were made that $\alpha=0, \gamma$ is not necessarily zero, coupling is direct and not diffusional. The authors were particular about studying effects caused by the non-isochronity of oscillators. In contrast, we do not make an isolated system possess complex dynamics but consider coupling of a good deal of generality.

In our studies we analyze the present system in detail for all $\alpha, \beta, \gamma$ and $\Delta$. Having numerically found the solutions of (5) (i.e. rest states of (4)), we determine their stability computing the correspondent eigenvalues using (4), thus deriving, which type of the region the concrete parameter values correspond to. Moreover, we focus our attention on the dependence of the phase, frequency and amplitude concerning synchronization on the parameter values. These characteristics are apparently very important ones, all the while they have not been considered in Refs. [15-19]. 


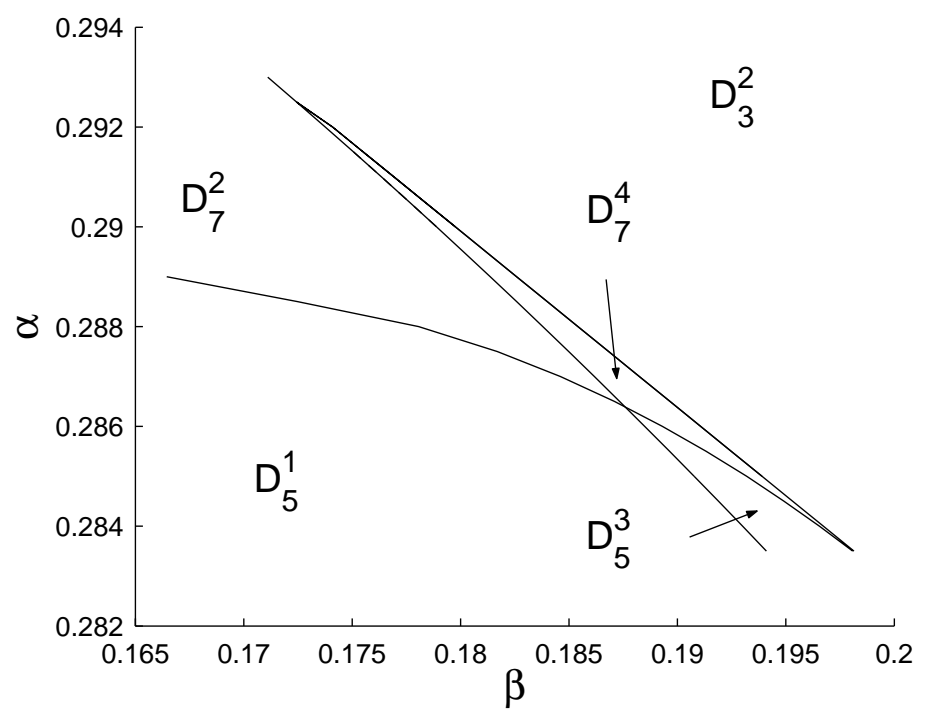

Fig. 5. Part of the bifurcation diagram on the right to the maximum of the curve $\alpha^{2}=\beta-3 \beta^{2}$ (Fig. 4(b)), here $\Delta=0, \gamma=0$. The upper indexes "1", "2", "3" and "4" correspond to the number of stable synchronization regimes.

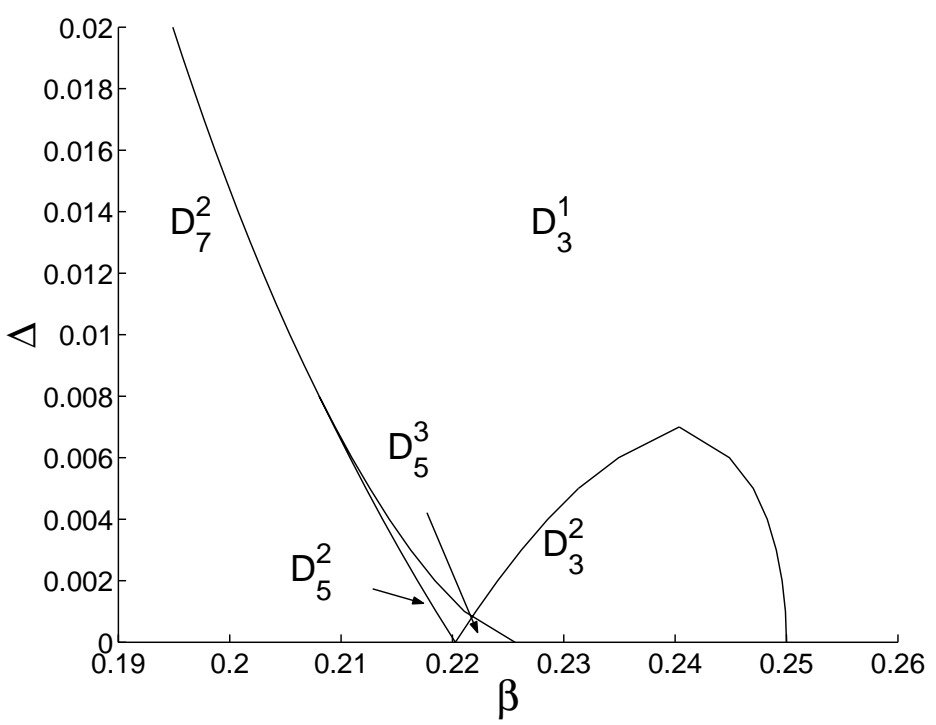

Fig. 6. Part of bifurcation diagram for $\alpha=0.27, \gamma=0$ illustrating the changes in multistability regimes non-zero $\Delta$ leads to. The upper indexes " 1 ", " 2 " and " 3 " correspond to the number of stable synchronization regimes.

\section{The case of non-zero frequency mismatch}

To begin with, we assume that $\gamma=0$ and gradually increase conservative coupling $\alpha$ from zero. We illustrate the dynamics of the studied system by plotting bifurcation diagrams that we derive using numerical algorithms, in the $(\beta, \Delta)$-plane for a sequence of $\alpha$. 
Appearance of bistability and Andronov-Hopf bifurcation: removal of degeneration. The region of bistability appears near $\Delta=0, \beta=0$ and has the very form and size it should possess according to Ref. [16] (Fig. 4(a)). The global picture also changes qualitatively (Fig. 3(a)). Now the degenerated point $\beta=1, \Delta=2$ vanishes and the direct transition from the beating regime $\mathrm{D}_{3}^{0}$ to the oscillator death regime (at $\beta=1, \Delta \geq 2$ ) becomes impossible. The new way is $\mathrm{D}_{3}^{0} \rightarrow \mathrm{D}_{3}^{1} \rightarrow \mathrm{D}_{2}^{1} \rightarrow \mathrm{D}_{1}^{1}$ and it requires this sequence of bifurcations to happen. Besides, if dissipative coupling $(\beta)$ is strong enough, the transition from the in-phase synchronization regime to the beating one is realized through a supercritical Andronov-Hopf bifurcation: $\mathrm{D}_{3}^{1} \rightarrow \mathrm{D}_{3}^{0}$; for weak dissipative coupling $(\beta)$ a saddle-node bifurcation happens: $\mathrm{D}_{5}^{1} \rightarrow \mathrm{D}_{3}^{0}$.

Enhancing of bistability properties: special types of bistability. As said before, when $\alpha$ exceeds the threshold 1/4 a new region of bistability appears (Fig. 4(c), region $\mathrm{D}_{3}^{2}$ ). When $\alpha$ is increased further, the regions $\mathrm{D}_{7}^{2}$ and $\mathrm{D}_{3}^{2}$ extent and finally merge (Fig. 4(d)). The principal result of this is that now the stability of the anti-phase synchronization regime can be lost not only through a saddle-node bifurcation but also as a result of a supercritical Andronov-Hopf bifurcation. It reveals the existence of qualitatively new effects, namely the bistability of a rest state and a limit cycle or that of two limit cycles of (1).

Andronov-Hopf bifurcation takes over the system. Finally, when the conservative coupling $\alpha$ turns strong enough, the bifurcation diagram becomes far less complex (Fig. 3(d)). We would like to note especially that the saddle-node bifurcation does not govern the system any more; each change occurring due to the supercritical Andronov-Hopf bifurcation in (4) $\left(\mathrm{D}_{3}^{2} \rightarrow \mathrm{D}_{3}^{1}, \mathrm{D}_{3}^{1} \rightarrow \mathrm{D}_{3}^{0}\right)$ or in (1) $\left(\mathrm{D}_{3}^{1} \rightarrow \mathrm{D}_{2}^{1}, \mathrm{D}_{2}^{1} \rightarrow \mathrm{D}_{1}^{1}\right)$. $\mathrm{D}_{3}^{2} \rightarrow \mathrm{D}_{3}^{1}$ leads to the coexisting of a stable rest state and a stable limit cycle, $\mathrm{D}_{3}^{1} \rightarrow \mathrm{D}_{3}^{0}$ leads to bistability of limit cycles (or beating regimes in (1)).

Multistability regimes. Let us now turn back to Fig. 4(b) and increase $\beta$ gradually, while $\alpha$ remains constant, $1 / 4<\alpha<1 / 2 \sqrt{3}$ and $\Delta=0$. According to Ref. [18], transitions across the parabolic line ${ }^{\pi} \mathrm{S} \leftrightarrow{ }^{\pi} \mathrm{U}$ (in ${ }^{\pi} \mathrm{S}$ the exact anti-phase rest state $(\bar{\phi}=\pi)$ is stable, in ${ }^{\pi} \mathrm{U}$ it is unstable) correspond to pitchfork bifurcation that involves the anti-phase synchronization regime. Obviously, it is in perfect agreement with Fig. 4(c) when the anti-phase solution loses its stability as $\mathrm{D}_{7}^{2} \rightarrow \mathrm{D}_{5}^{1}$, but a direct transition $\mathrm{D}_{5}^{1} \rightarrow \mathrm{D}_{3}^{2}$ through the pitchfork bifurcation is impossible. A detailed analysis reveals utterly amazing results. The part of the bifurcation diagram on the right to the maximum of the curve $\alpha^{2}=\beta-3 \beta^{2}$ (Fig. 4(b)) is presented in Fig. 5. As long as two simultaneous Andronov-Hopf bifurcations occur in transitions $\mathrm{D}_{5}^{1} \rightarrow \mathrm{D}_{5}^{3}, \mathrm{D}_{7}^{2} \rightarrow \mathrm{D}_{7}^{4}$, regions, where three and four stable solutions coexist, appear (new stable solutions are anti-phase). Apparently, going backwards $\mathrm{D}_{5}^{3} \rightarrow \mathrm{D}_{5}^{1}$ or $\mathrm{D}_{7}^{4} \rightarrow \mathrm{D}_{7}^{2}$ we find ourselves in the regions of a special kind of multistability: two limit cycles and one or two rest states, respectively, are simultaneously stable. Our results show that $\Delta \neq 0$ makes these regions vanishing rapidly and violates simultaneousness two Andronov-Hopf bifurcations (Fig. 6).

As long as stable non-zero rest states are of special interest in applications, we would like to summarize the results, described above and present synchronization regions for different values of conservative coupling $\alpha$ (Fig. 7). There, the evolution of the in-phase synchronization and bistability regimes regions is shown. The region, where at least one synchronization regime exists, is marked S (Fig. 7(a)) and lies beneath the bifurcation curves that separate it from the beating regime region $\mathrm{B}$ (to the left) and the oscillator death region OD (to the right). This comparison clearly shows that the increase of the strength of conservative coupling makes the synchronization region growing at the expanse of the oscillator death and the beating ones. According to the presented results, an optimal strength of dissipative coupling can be claimed to exist. Here synchronization holds for an arbitrary large frequency mismatch and the "channel" around the asymptote $\beta=1$ broadens as conservative coupling is increased. At the same time, the region, where only one stable (in-phase) synchronization regime exists $\left({ }^{0} \mathrm{~S}^{1}\right.$, Fig. $\left.7(\mathrm{~b})\right)$, is partially substituted by the region of bistability $\mathrm{S}^{2}$. The latter is situated under the bifurcation curves (for different $\alpha$, respectively) that divide it from the former one. Note that while the height of $S^{2}$ gradually increases, its width cannot exceed $\beta=1 / 4$. 

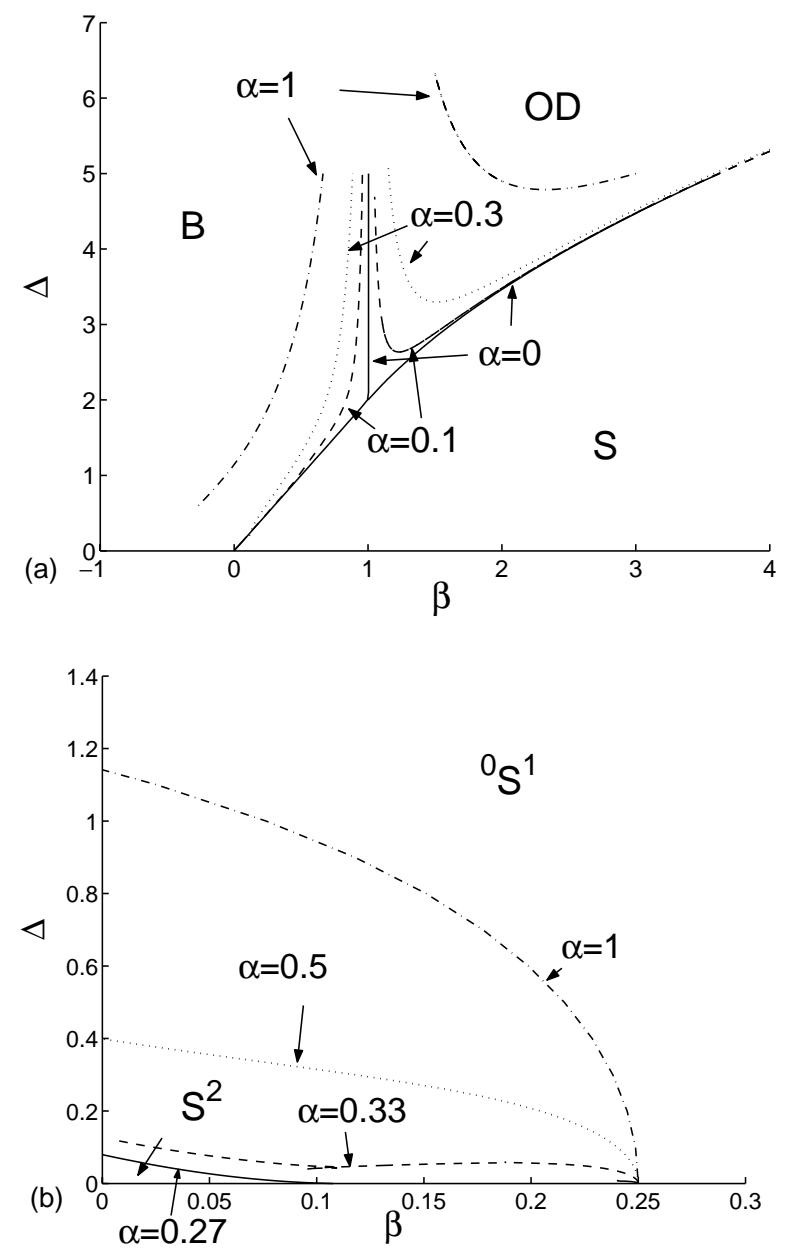

Fig. 7. (a) Boundaries of the regions of synchronization (marked by $\mathrm{S}$, for each value of $\alpha$ the region lies under the bifurcation curves) the beating regime region is marked by $\mathrm{B}$, the "oscillator death" region is marked by OD. (b) Boundaries of the bistability regime (for each value of $\alpha$ the bistability region lies under the bifurcation curves and is marked $\mathrm{S}^{2}$, the region of monostability is marked by ${ }^{0} \mathrm{~S}^{1}$ (the left upper index refers to the in-phase regime: $|\bar{\phi}| \approx 0)$ ).

\section{Characteristics of synchronization regimes}

Now we study the frequency and the amplitude characteristics of the synchronization regimes existing in our system.

If $\alpha, \beta \ll 1$, then using propositions discussed in Section 3, one can easily get the frequency synchronization

$$
\omega_{\mathrm{s}}=-\dot{\phi}_{1}=-\frac{\Delta}{2}-\alpha(1-\cos \bar{\phi})
$$

(the frequency, observed in the system (1) is $\left.\tilde{\omega}_{\mathrm{s}}=1+\mu \omega_{\mathrm{s}} / 2\right)$ neglecting terms of $\mathrm{O}(\alpha \beta)$.

According to (9), the in-phase solution implies synchronization has the mean frequency

$$
\omega_{\mathrm{s}}=-\dot{\phi}_{1}=-\dot{\phi}_{2} \approx \frac{\Delta}{2}
$$


and that of the anti-phase solution the synchronization frequency is

$$
\omega_{\mathrm{s}}=-\dot{\phi}_{1}=-\dot{\phi}_{2} \approx 2 \alpha+\frac{\Delta}{2} \text {. }
$$

Numerical simulations show that there is no qualitative difference in the case when $\alpha$ and $\beta$ are not small (Fig. 8(a)(d)). This leads to the conclusion that the phase, frequency and amplitude of synchronization remain qualitatively the same in a broad range of parameter values, and show deviations only near a saddle-node bifurcation. In particular, the synchronization frequency is also in a quite good quantitative agreement with (10) and (11).

It turns out that additional synchronization regimes that provide multistability in the system (see regions $\mathrm{D}_{5}^{3}$ and $\mathrm{D}_{7}^{4}$ in Figs. 5 and 6), do not contribute qualitatively new frequency values (Fig. 9(a) and (b)). In fact, they remain stable only in a narrow region, when they are situated near anti-phase solution, as considered above. Being in this vicinity, they posses some identical characteristics, namely, they are also anti-phase solutions, although, the changes in the amplitude values are quite distinct and give an illustration of the pitchfork bifurcation, taking place in the system (4) (symmetry breaking bifurcation of stable limit cycle in the original system (1)).

Frequency asymmetry. Now let us turn to the area between the beating regime and the oscillator death regions with characteristic value of $\beta$ near 1 (Fig. 7(a)) that we have described before as an optimal coupling value. The correspondent boundary curves have an asymptote $\beta=1$, the width of this region decreases to zero for $\Delta \rightarrow \infty$. Increasing gradually $\Delta$, we get the following numerical result: the synchronization properties change dramatically and a new regime appears. The frequency of synchronization deviates from the mean frequency and approaches that of the first oscillator (Fig. 10(c)), which equals

$$
\omega_{\mathrm{s}}=\alpha .
$$

At the same time its amplitude exceeds the amplitude of the second one substantially, showing finally an almost complete dominance $\left(\bar{R}_{1} \gg \bar{R}_{2}\right)$ (see Fig. 10(a)). Besides the phase difference of synchronization seems to approach some limit value (Fig. 10(b)). The comparison shows that the difference of the synchronization frequency from that of the isolated first oscillator $\left|\omega_{\mathrm{S}}-\alpha\right|$ and the rate of the amplitudes $1 / r=R_{2} / R_{1}$ are equally small, while approaching limit values (Fig. 10(d)).

This result deserves a detailed consideration as it claims the existence of a principle frequency asymmetry induced by a non-zero $\Delta$. In spite of the fact that $\mu \Delta$ is always small, which naturally leads to the assumption of oscillator's equality with respect to frequency values, the following situation takes place: the smaller frequency turns to be preferred for a large frequency mismatch $\Delta$, when $\beta$ is too big to allow the beating regime exist, though not big enough to cause oscillator death.

In order to prove this result, we seek the solution of (5) in the form

$$
\bar{R}_{2}=\frac{\bar{R}_{1}}{r}
$$

where $r \gg 1$ (i.e. $\bar{R}_{1} \gg \bar{R}_{2}$ ). Then, neglecting terms of $\mathrm{O}\left(1 / r^{2}\right)$, we simplify (5) to the following system:

$$
\begin{aligned}
& \bar{R}_{1}\left(1-\beta-\frac{1}{4} \bar{R}_{1}^{2}\right)+\bar{R}_{2}(\alpha \sin \bar{\phi}+\beta \cos \bar{\phi})=0, \quad \bar{R}_{2}\left(1-\beta-\frac{1}{4} \bar{R}_{2}^{2}\right)+\bar{R}_{1}(-\alpha \sin \bar{\phi}+\beta \cos \bar{\phi})=0, \\
& \Delta-(\alpha \cos \bar{\phi}+\beta \sin \bar{\phi}) r=0 .
\end{aligned}
$$

Using (13) we get

$$
\begin{aligned}
& r\left(1-\beta-\frac{1}{4} \bar{R}_{1}^{2}\right)+\alpha \sin \bar{\phi}+\beta \cos \bar{\phi}=0, \quad 1-\beta-\frac{\bar{R}_{1}^{2}}{4 r^{2}}+r(-\alpha \sin \bar{\phi}+\beta \cos \bar{\phi})=0, \\
& r=\frac{\Delta}{\alpha \cos \bar{\phi}+\beta \sin \bar{\phi}} .
\end{aligned}
$$



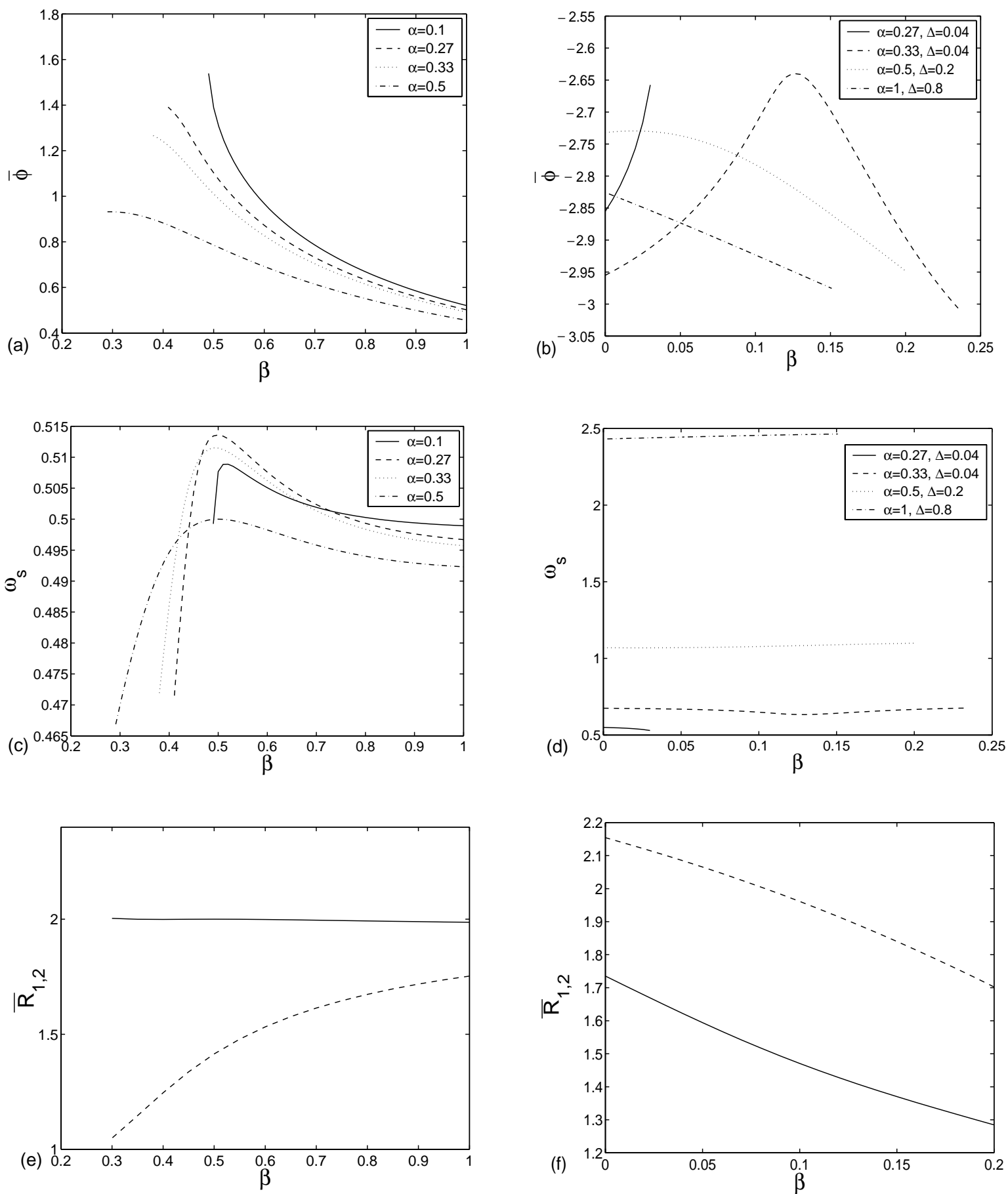

Fig. 8. Phase difference, synchronization frequency and amplitude characteristics of the in- and anti-phase solutions: (a) and (c) stable in-phase solution for fixed $\Delta=1$; (b) and (d) stable anti-phase solution, typical amplitude values for: (e) the in-phase $(\alpha=0.5, \Delta=1)$; (f) anti-phase ( $\alpha=0.5, \Delta=0.2)$ synchronization regimes $\left(R_{1}\right.$, solid lines; $R_{2}$, dashed lines). 

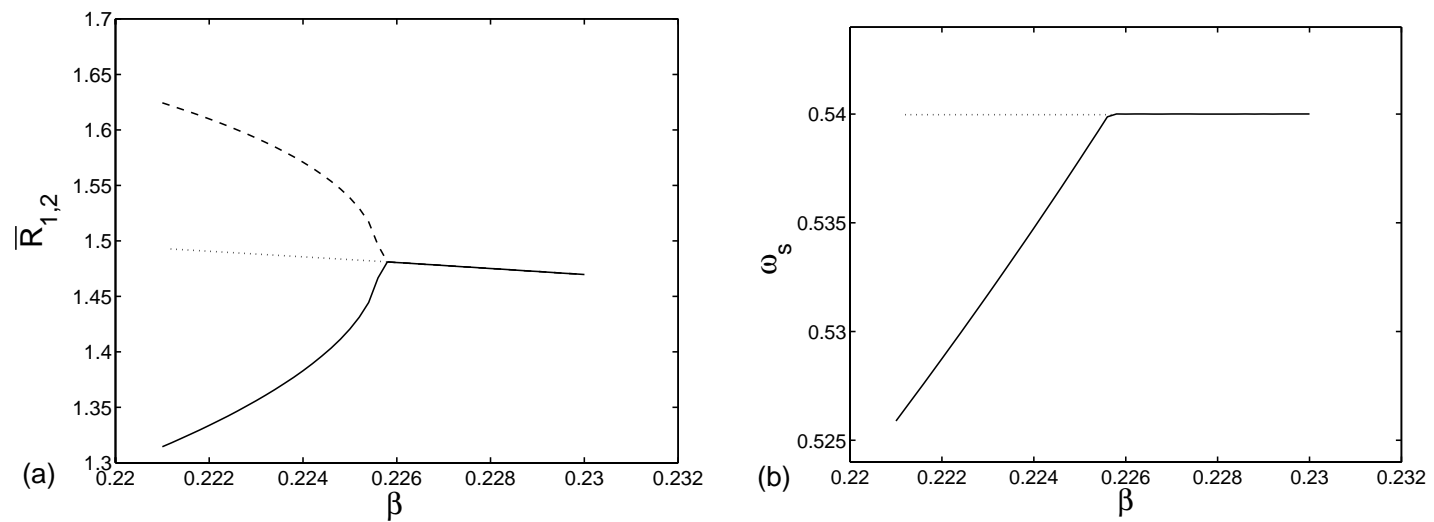

Fig. 9. Amplitude and frequency characteristics of the regimes that provide multistability $(\alpha=0.27, \gamma=0, \Delta=0)$ : (a) amplitude values (solid and dashed lines mark $\bar{R}_{1}$ and $\bar{R}_{2}$, respectively, for one of the additional stable solutions and vice versa for the other, the solutions being symmetric: $\bar{R}_{1} \rightarrow \bar{R}_{2}, \bar{R}_{2} \rightarrow \bar{R}_{1}, \bar{\phi} \rightarrow \pi-\bar{\phi}$ ); (b) solid line marks the frequency values of the additional regimes, which are equal; dotted line marks the pure anti-phase solution $(\bar{\phi}=\pi)$ while it is unstable.
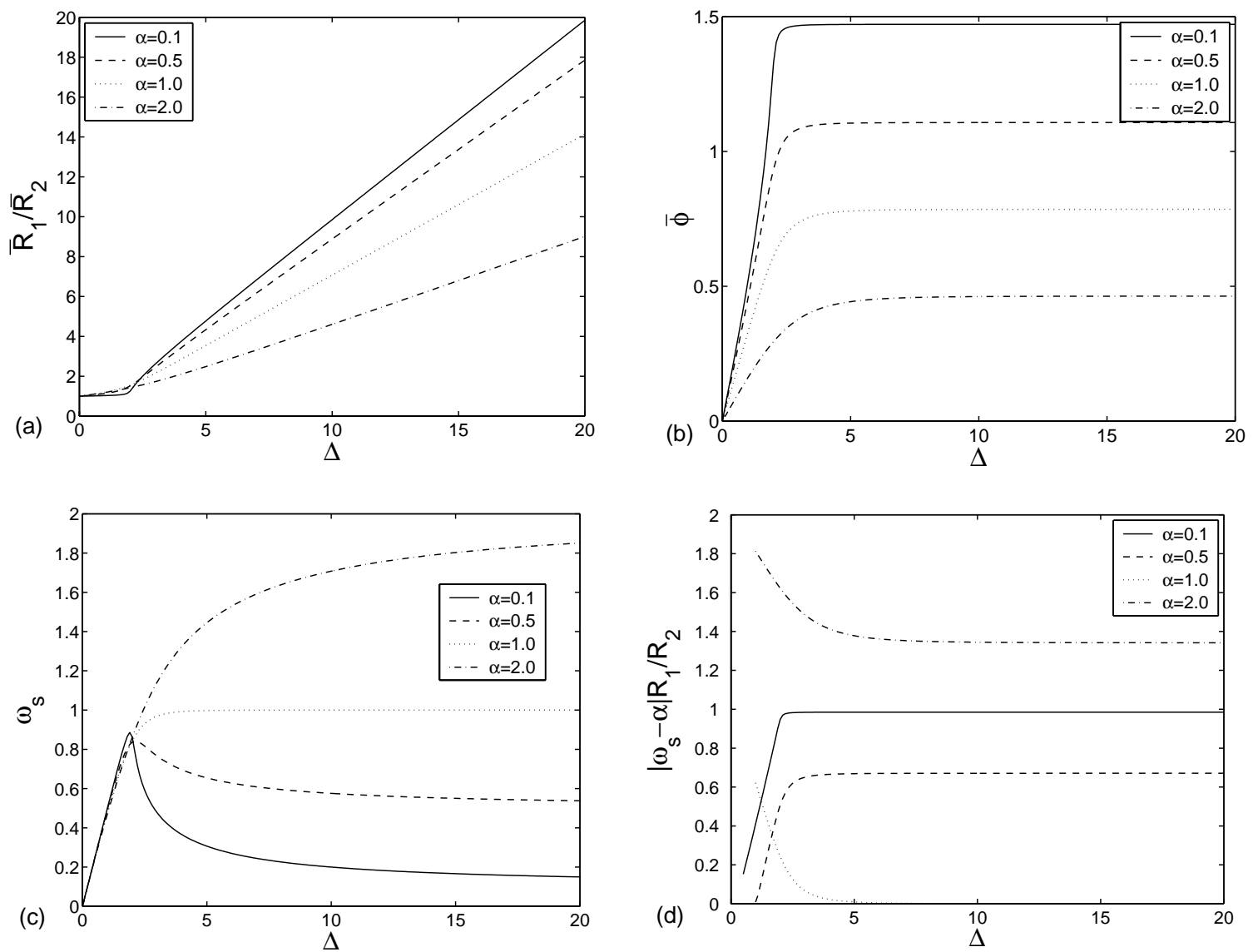

Fig. 10. Amplitude (a), phase (b) and frequency (c) characteristics of the synchronization regime and the proportion of frequency deviation from $\alpha$ to the rate of amplitudes $\left|\omega_{\mathrm{s}}-\alpha\right|\left(R_{1} / R_{2}\right)$ (d) for fixed $\beta=1, \gamma=0$. 
Let $\beta=1$, then from the first equation $r\left(\bar{R}_{1}^{2} / 4\right) \leq \sqrt{\alpha^{2}+1}$, consequently $\bar{R}_{1}^{2} / 4 r^{2} \ll 1$. This yields

$$
\bar{R}_{1}=2 \sqrt{\frac{2 \alpha}{\Delta}}, \quad r=\frac{\Delta}{\sqrt{\alpha^{2}+1}}, \quad \tan \bar{\phi}=\frac{1}{\alpha}, \quad\left(\omega_{2}\right)_{\mathrm{s}}=-\dot{\varphi}_{2}=\alpha .
$$

Evidently, the bigger $\Delta$, the more precisely $r \gg 1$ is obtained, and the better our approximate solution fits the original system and the closer to $\alpha$ the frequency of synchronization is.

Transition to the beating regime. Another important characteristic of interacting systems, where synchronization can take place, is the averaged beating frequency. Its definition is

$$
\omega_{\mathrm{b}}=\lim _{T \rightarrow \infty} \frac{1}{T} \int_{0}^{T} \dot{\phi} \mathrm{d} t=\lim _{T \rightarrow \infty} \frac{\phi(T)-\phi(0)}{T} .
$$

Three types of qualitatively different behavior may be observed in this system:

- $\phi=\varphi_{1}-\varphi_{2}=$ const. corresponds to the synchronization regime, $\omega_{\mathrm{b}}=0$.

- $|\phi|=\left|\varphi_{1}-\varphi_{2}\right| \leq$ const. means that a stable limit cycle exists, and changes in the phase difference of the solution are limited (it is called "phase entrainment" [24] or "phase trapping" [18]); $\omega_{\mathrm{b}}$ still equals zero.

- Finally, if $|\phi|=\left|\varphi_{1}-\varphi_{2}\right|$ grows unbounded, $\omega_{\mathrm{b}} \neq 0$ now, then a stable limit cycle (also called a "libration orbit" [24]) corresponds to a phase drift regime.

The transition between the second and the third types of behavior was the subject of study in Ref. [24] for the special case $\alpha \neq 0, \Delta \neq 0, \beta=\gamma=0$.

In the general case, which we are dealing with in the present paper, each of the possible transitions has been analyzed. We have investigated the ways of losing synchronization that appear in this system obtaining numerically (performing simulation of the original system (1) for $\mu=0.1$ ) the averaged beating frequency $\omega_{\mathrm{b}}$ for $\gamma=0$, changing $\Delta$, a fixed $\alpha$, and a sequence of $\beta$ (Fig. 11(a)) and vice versa (Fig. 11(b)). These diagrams present visual information about the behavior of the system. Indeed, when a stable rest state disappears through a saddle-node bifurcation, a stable limit cycle with a phase difference growing unbounded is born instead. As long as the flow slows down near the location of the former rest state, the period of this cycle is very big near the bifurcation point, making the averaged beating frequency increasing continuously (Fig. 11(a)), i.e. a "soft" transition to synchronization takes place. Nevertheless, the bigger $\beta$, the sharper the slope of the frequency curve is. Quite the opposite scenario is
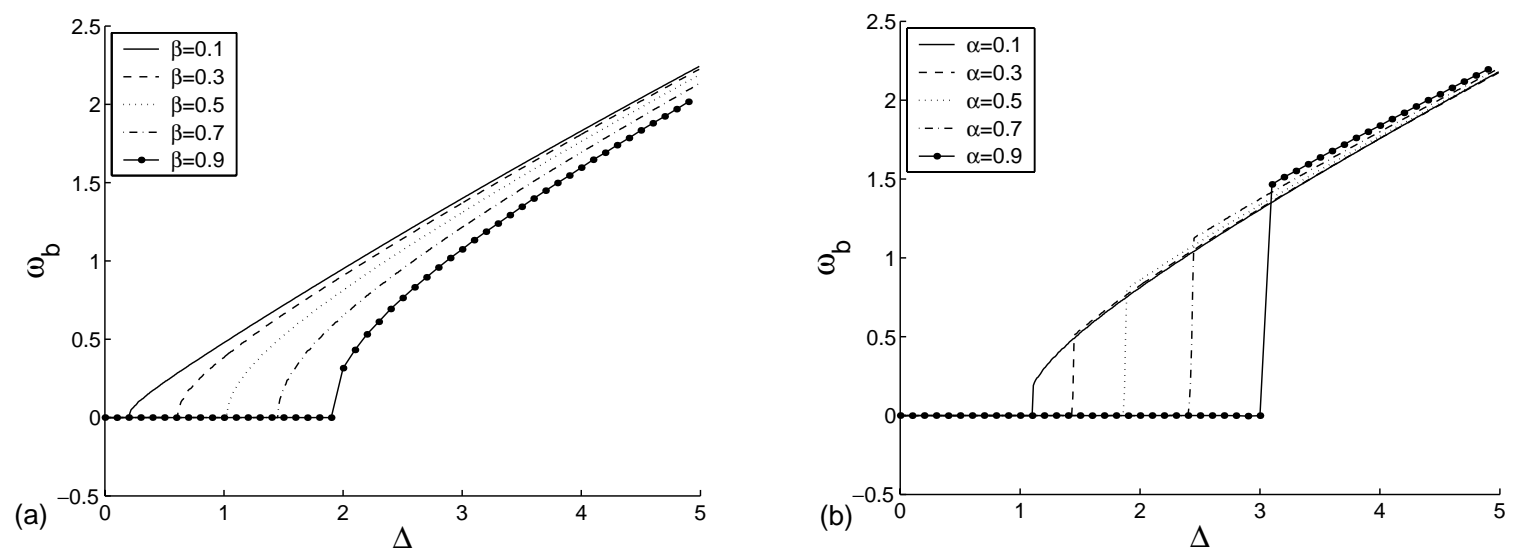

Fig. 11. Averaged beating frequency calculated in original system (1) for $\mu=0.1$ in case $\gamma=0$ illustrates soft and hard transitions from the synchronous to the beating regime: (a) fixed $\alpha=0$; (b) fixed $\beta=0.5$. 
observed, when an Andronov-Hopf bifurcation takes place. A limit cycle with a bounded variation of the phase difference appears and it requires bigger frequency mismatches $\Delta$ to show a non-zero frequency (Fig. 11(b)), i.e. a "hard" transition is observed. When the phase of the solution becomes eventually drifting, the period is far from being infinitely big. Consequently, the discontinuity of the frequency characteristic corresponds to a manifold in the parameter space. By crossing of that the beating regime distinctively changes its properties giving rise to the limit cycle with unbounded phase growth (drift).

\section{Oscillators with frequency and amplitude mismatches and their response characteristics}

In contrast to the previous sections we do not separate description of bifurcations that take place in the system and that of the amplitude, phase difference and synchronization frequency characteristics. The reason is that in the case we are dealing with now, these characteristics provide a better understanding of bifurcations in the system, and sometimes they are absolutely necessary for it.

So, we remove the assumption that $\gamma=0$ and study changes in the bifurcation portraits and effects which follow.

Monostability of the anti-phase synchronization regime. The first effect we have found is the appearance of the region where the only stable solution is the anti-phase rest state (Fig. 12, regions ${ }^{\pi} D_{5}^{1},{ }^{\pi} D_{3}^{1}$ ) that enlarges at the expense of the beating and the bistability regions as $\gamma$ is increased.

For $\alpha, \beta \ll 1$ and $\gamma \ll 1$ this result can be derived analytically by using the same technique as in Ref. [16]. We would not go into details here and instead present numerical results which show the region of the anti-phase synchronization for different values of conservative coupling (Fig. 12).

Disappearance of bi- and multistability. When $\gamma$ is increased further, the region of bistability $\left(\mathrm{D}_{7}^{2}, \mathrm{D}_{3}^{2}\right.$, Fig. 12(a) and (b)) diminishes and finally disappears. After that no further qualitative change in this part of the bifurcation diagram is observed (Fig. 13(a)). In addition we have found all multistability regimes reported above to vanish rapidly as $\gamma$ becomes non-zero. Hence, $\gamma \neq 0$ tends to eliminate every bi- and multistability, which otherwise is possible.

Unusual loss of synchronization and further reentry. It is important to note that as long as $\gamma$ is not small, synchronization changes its characteristic from anti-phase to in-phase continuously, i.e. it undergoes no bifurcation
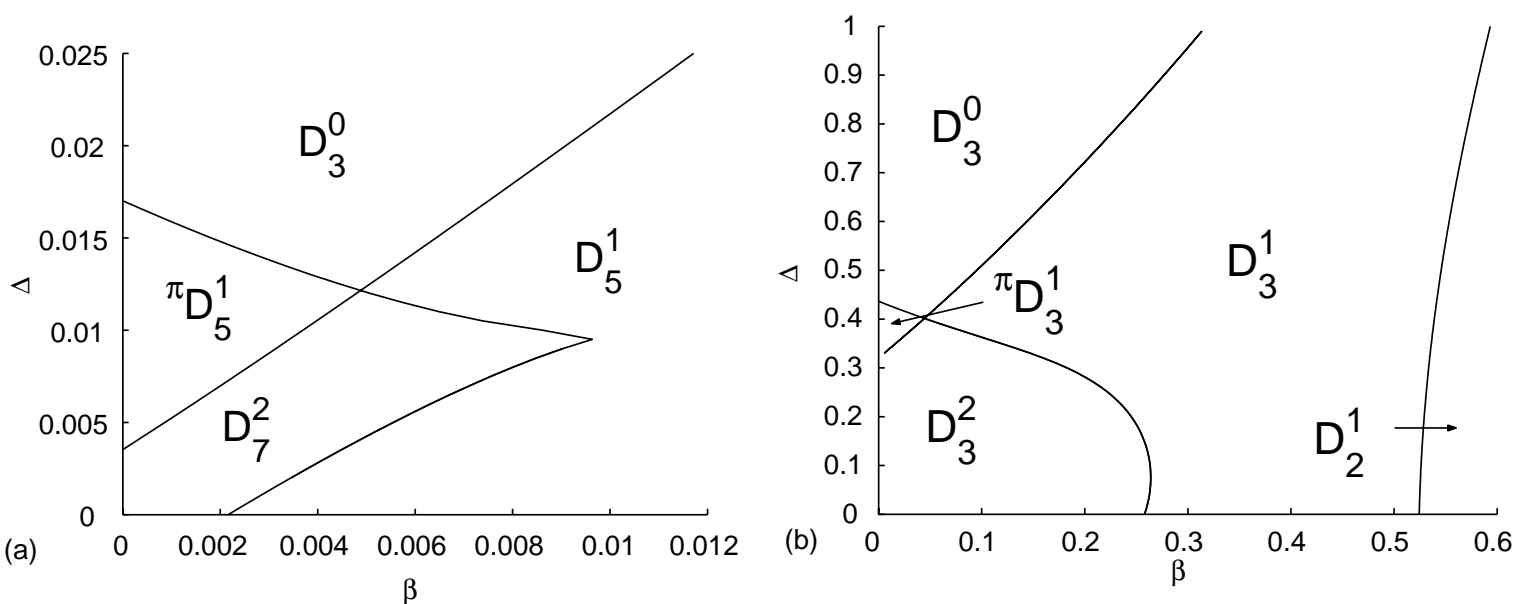

Fig. 12. (a) Part of bifurcation diagram for $\alpha=0.1, \gamma=0.1\left(\mathrm{D}_{2}^{1}\right.$ and $\mathrm{D}_{1}^{1}$ are not shown here); (b) part of the bifurcation diagram for $\alpha=0.5$, $\gamma=0.1$ ( $\mathrm{D}_{1}^{1}$ is not shown here). In the regions with the right upper indexes " 1 " and " 2 " mono- and bistability takes place, respectively. The left upper index " $\pi$ " refers to the anti-phase synchronization. As before, $\mathrm{D}_{3}^{0}$ corresponds to the beating regime. 

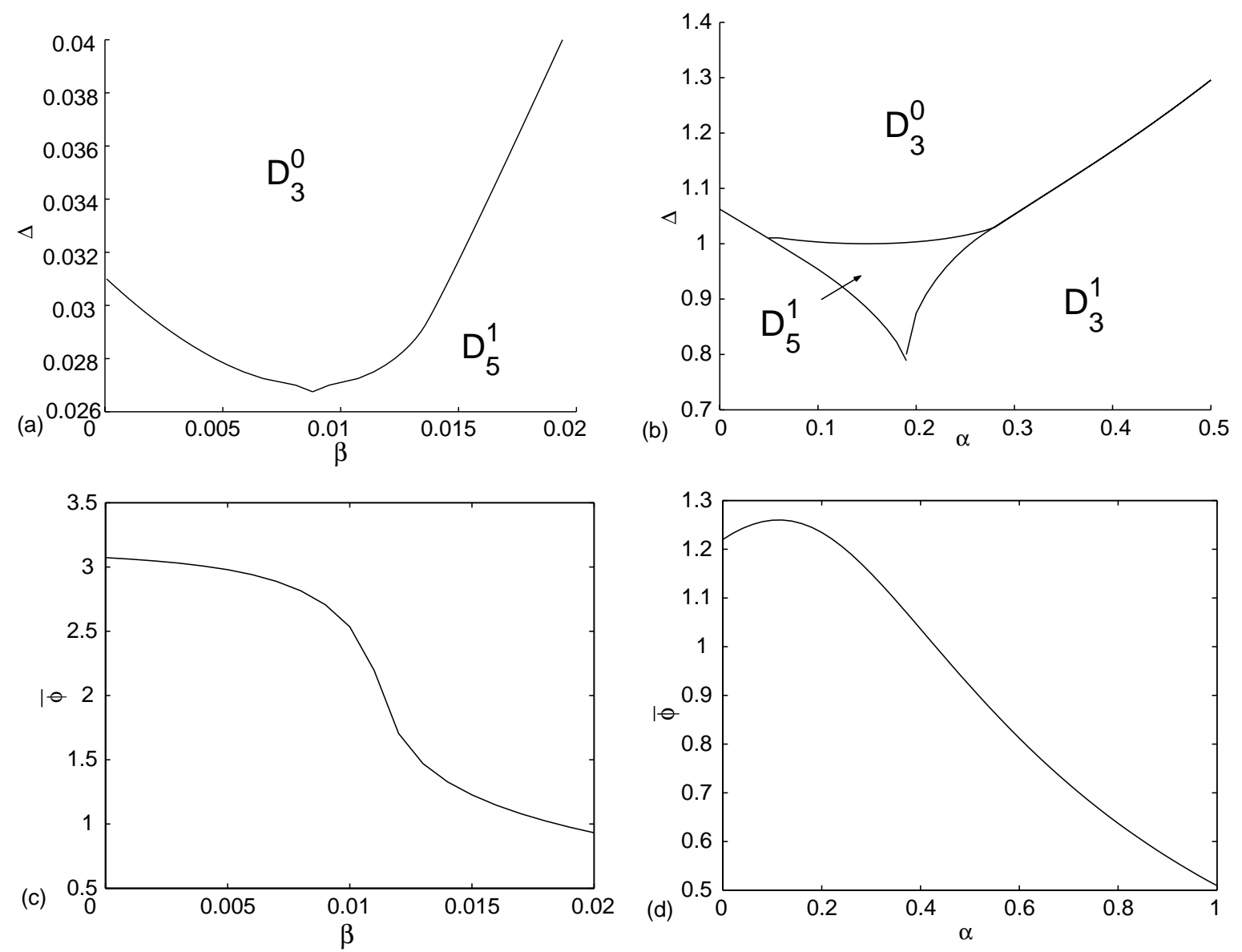

Fig. 13. (i) Part of bifurcation diagram for: (a) $\alpha=0.1, \gamma=0.3$; (b) $\beta=0.5, \gamma=0.3$ ( $\mathrm{D}_{3}^{1}$, $\mathrm{D}_{2}^{1}$ and $\mathrm{D}_{1}^{1}$ are not shown here); (ii) phase difference values illustrating continuous transition from anti- to in-phase synchronization regime for $\alpha=0.1, \gamma=0.3, \Delta=0.025$ (c); (iii) phase difference changes inside the in-phase synchronization region that corresponds to case (b) for $\beta=0.5, \gamma=0.3, \Delta=0.7$ (d).

as the parameters are being changed (Fig. 13(a) and (c)). This is in contrast to the case of $\gamma=0$ (Sections 4 and 5). Besides, one can easily notice another peculiarity introduced by $\gamma \neq 0$ : when $\Delta$ belongs to a certain range, an increase of each of the coupling parameters $(\alpha$ or $\beta)$ makes synchronization disappear $\left(\mathrm{D}_{5}^{1} \rightarrow \mathrm{D}_{3}^{0}\right.$, Fig. 13(a) and $D_{3}^{1} \rightarrow D_{3}^{0}$, Fig. 13(b)) and then after a while appear again $\left(D_{3}^{0} \rightarrow D_{5}^{1}\right.$, Fig. 13(a) and $D_{3}^{0} \rightarrow D_{3}^{1}$, Fig. 13(b)). This seems most unusual as increasing liability to synchronization in the system used to be firmly associated with the increase of $\alpha$ and $\beta$. It may either be followed by qualitative change in the phase difference (from the anti-phase to the in-phase as $\beta$ is changed, Fig. 13(c)) or not (remaining the in-phase one as $\alpha$ is changed, Fig. 13(d)).

"Oscillator death" and frequency pull-in effect analog. Let us turn now to the stable origin region and its neighbor regions. We mainly show that the effect of "oscillator death" still remains, in case of non-zero $\gamma$. But the region $\mathrm{D}_{2}^{1}$, where the non-zero steady state of (4) is stable and the zero rest state is unstable, enlarges at the expense of the "oscillator death" region and forms an additional region (Fig. 14, compare with Fig. 7(a)). The latter was found to exist for arbitrary $\alpha$ and $\gamma$. The bigger $\alpha$ and $\gamma$, the broader along $\beta$ this region is and the smaller is the minimum value of $\Delta$ for this region to begin. If $\gamma \leq 2 \alpha$ there exists a degenerated point $\mathrm{A}$ that belongs to the bifurcation curves 


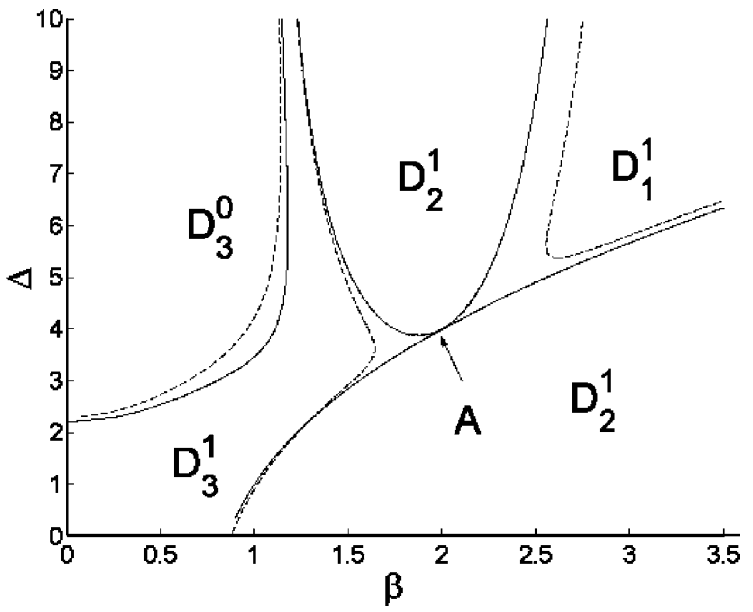

Fig. 14. Bifurcation diagrams for $\alpha=1, \gamma=2$ (solid lines), and $\gamma=2.2$ (dashed lines).

between the regions $D_{1}^{1}, D_{2}^{1}$ and $D_{3}^{1}$ (Fig. 14, solid lines), otherwise for a certain range of $\beta$ this regime persists for arbitrary $\Delta$ (Fig. 14, dashed lines). In addition we study the changes in the bifurcation curves for negative values of $\Delta$ and find no qualitative difference in that case except the following one: the degenerated point mentioned above (Fig. 14, solid lines) does not exist for any $\alpha$ and $\beta$ and the bifurcation diagram is equivalent to that in Fig. 14 marked with dashed lines.

As existence and position of this degenerated point defines the configuration of the regions in the parameter plane, it is reasonable to carry out a linear stability analysis of the zero rest state of (2).

Evidently, the degenerated point corresponds to a bifurcation, whose codimension is 2, namely, two limit cycles, the stable and the saddle ones, which merge with the origin simultaneously (in system (1)). As long as two conjugated eigenvalues must have zero real parts in case of the Andronov-Hopf bifurcation, in our case all four eigenvalues of our four dimensional system must have their real parts also zero and be two conjugated pairs. Linearization of system (2) near the origin gives the following matrix:

$$
D=\left\|\begin{array}{cccc}
1-\beta & -\alpha & \beta & \alpha \\
\alpha & 1-\beta & -\alpha & \beta \\
\beta & \alpha & 1+\gamma-\beta & -\Delta-\alpha \\
-\alpha & \beta & \Delta+\alpha & 1+\gamma-\beta
\end{array}\right\| .
$$

On completing tedious algebraic manipulations (searching the eigenvalues of the matrix $D$ ), we obtain conditions for the existence of degenerated point and its coordinates in the $(\beta, \Delta)$ parameter plane, respectively,

$$
2 \alpha>\gamma, \quad \beta=1+\frac{\gamma}{2}, \quad \Delta=2 \alpha\left(1+\frac{2}{\gamma}\right) .
$$

One can easily see that these results are in perfect agreement with the numerical ones. The simplicity of the form is the advantage one cannot underestimate, as these terms clearly show that the height of the degenerated point, which is the lower boundary of the additional region, turns to infinity as $\gamma \rightarrow 0$ and that when $\gamma>2 \alpha$ this point does not exist.

In order to understand this effect better, we have studied changes in the oscillators amplitudes and frequency of synchronization for $\alpha, \beta, \gamma$ fixed and $\Delta$ being increased from zero. The following has been revealed: for $\Delta$ big 

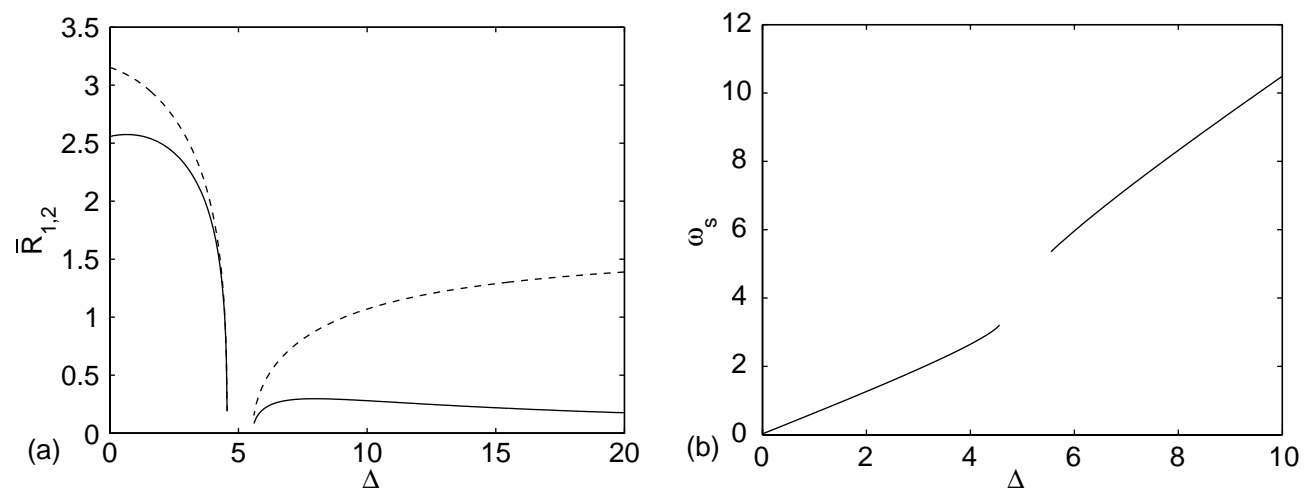

Fig. 15. (a) Amplitudes $\bar{R}_{1}$ (solid line), $\bar{R}_{2}$ (dashed line); (b) synchronization frequency $\omega_{\mathrm{s}}$ vs. $\Delta$ for fixed $\alpha=1, \gamma=2, \beta=2.3$ illustrate the dominance of the second oscillator. Note that synchronization frequency is near $\Delta / 2$ (mean frequency value), when oscillators demonstrate equality with respect to interaction and near $\Delta$ (dominating oscillator frequency value), when one of them dominates the other.

enough, the oscillators become qualitatively unequal and one of them (the amplitude of which when being isolated is bigger, however, may be just a little bigger) dominates the other one by lowering its amplitude down to zero as $\Delta \rightarrow \infty$, providing that synchronization lasts (Fig. 15(a) and (b)), and making the synchronization frequency close to the value that it demonstrates when isolated. The specialty of this effect is that the width of the region, where it persists, remains non-zero for arbitrary big $\Delta$, in contrast to the case we observed in the previous section $(\gamma=0)$ and now the second oscillator (that of the higher frequency) dominates, which was impossible before. So far, the effect that takes place in case $\gamma>0$ should by no means be regarded as some singular, non-robust one.

We would like to specially stress that to obtain prevalence of the second oscillator and, consequently, synchronization frequency being close to that of the isolated second oscillator the following has to be taken into account: there is no need for the amplitude of the first oscillator in case of uncoupled systems to be far smaller than that of
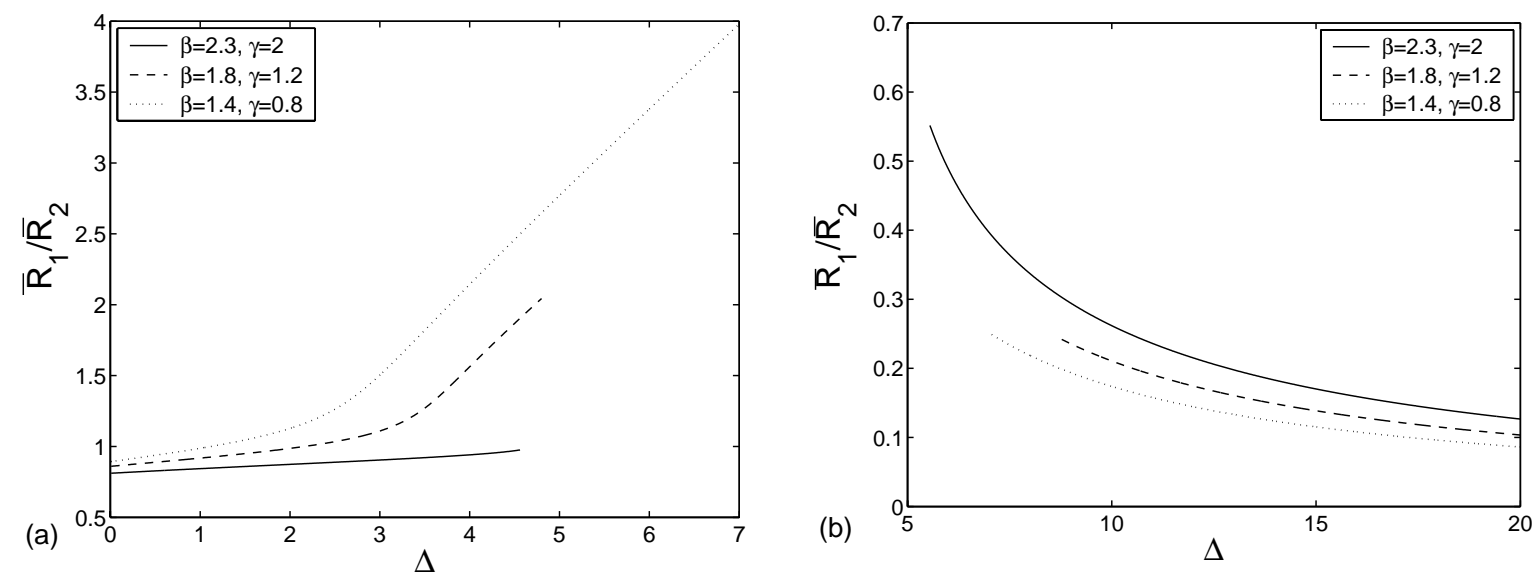

Fig. 16. For $\alpha=1$ we show typical cases of dominance of the first oscillator (under the degeneration point (19)) and of the second one (above the degeneration point (19)). 

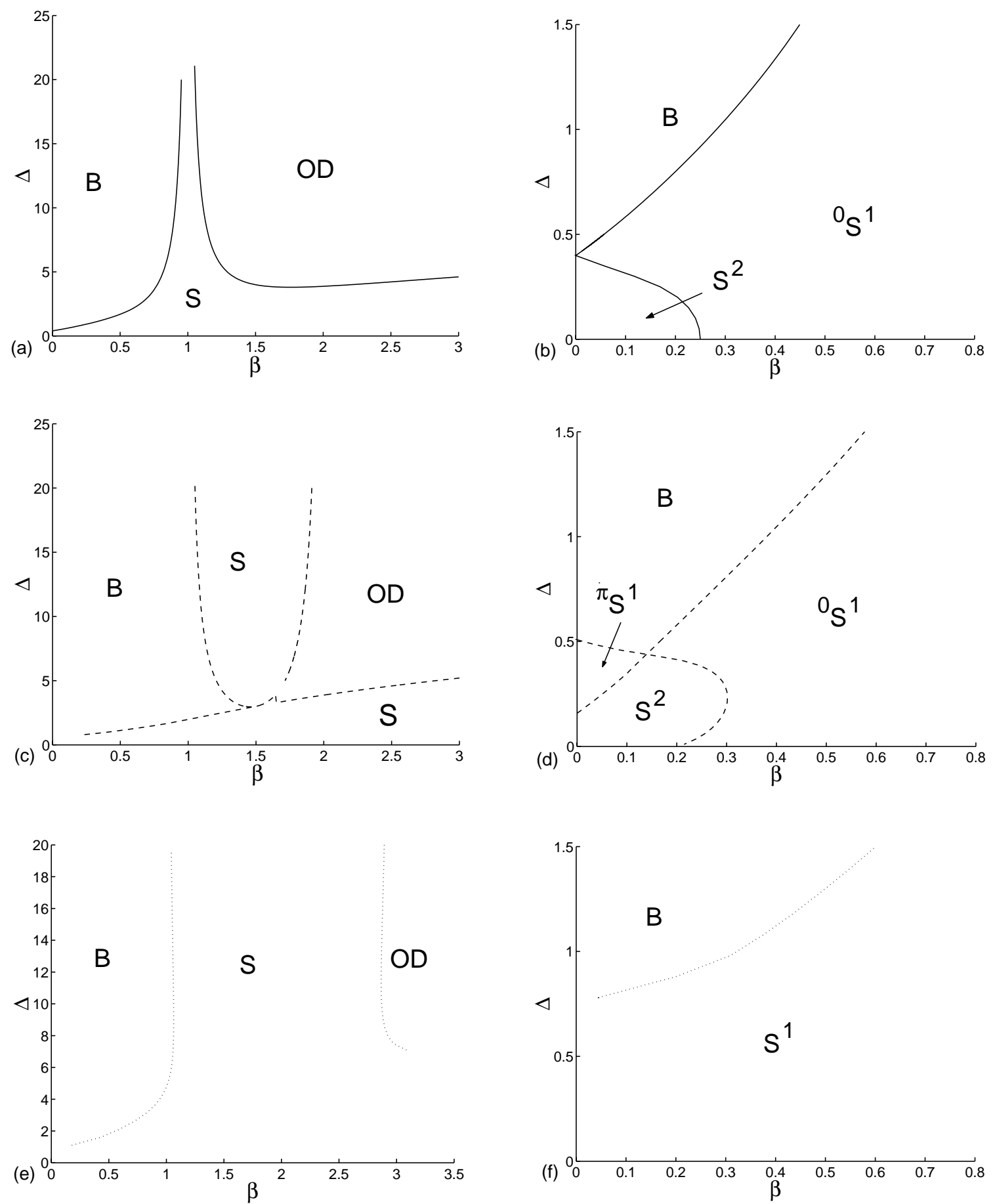

Fig. 17. (i) Regions of synchronization (S) for $\alpha=0.5$ and (a) $\gamma=0$, (c) $\gamma=1$, (e) $\gamma=2$; (ii) regions of bistability ( $\mathrm{S}^{2}$ ), surrounded by the in- $\left({ }^{0} \mathrm{~S}^{1}\right)$ and anti-phase $\left({ }^{\pi} \mathrm{S}^{1}\right)$ synchronization regime regions: $\alpha=0.5$ and (b) $\gamma=0$, (d) $\gamma=0.1$, (f) $\gamma=1.2$. The beating regime region is marked by B, the "oscillator death" regron is marked by OD. In (f) the symbol S ${ }^{1}$ denotes the region of the monostable synchronization. 

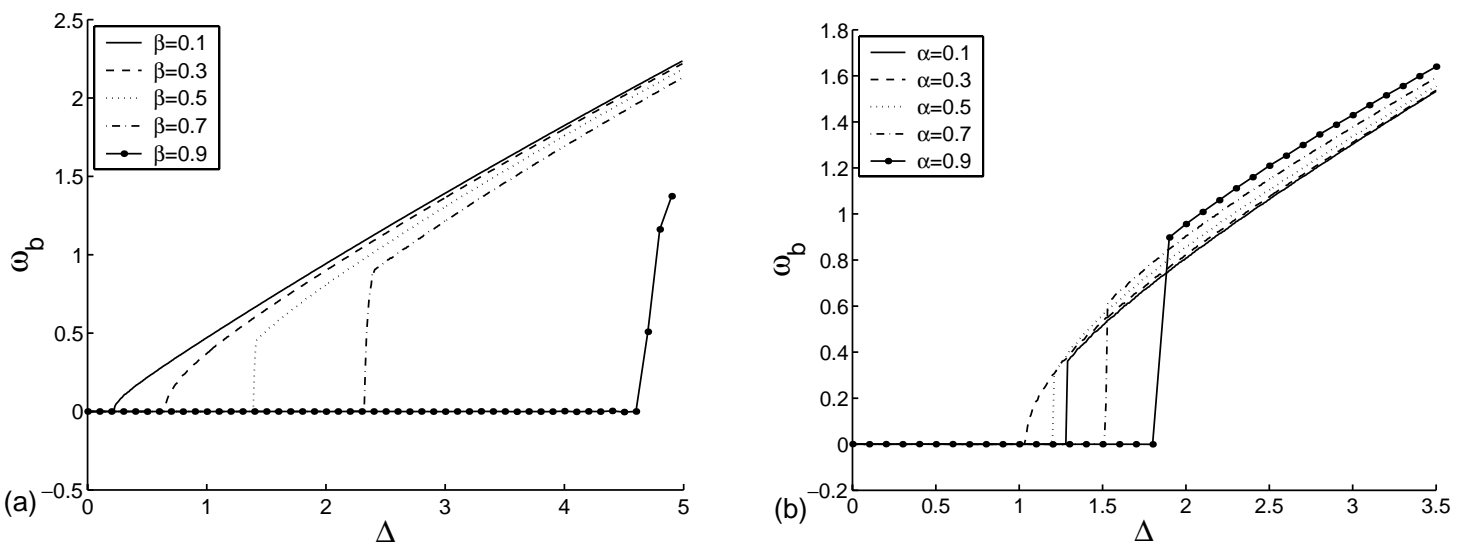

Fig. 18. Averaged beating frequency calculated in original system (1) for $\mu=0.1$ in case $\gamma=0.5$ : (a) fixed $\alpha=0$; (b) fixed $\beta=0.5$.

the second one, i.e. the limit of unidirectionally coupling is not necessary to provide it. Furthermore, according to considerations presented above, this effect takes place for every $\gamma \neq 0$, whatever small it is, in other words, for oscillators with arbitrary small difference in amplitude values when isolated.

The relation between the amplitudes $\bar{R}_{1} / \bar{R}_{2}$ vs. frequency mismatch $\Delta$ is shown in Fig. 16 for different values of the amplitude mismatch $\gamma$ and dissipative coupling $\beta$. One can see that for small $\gamma$ each oscillator can dominate the other one. In the region under the degenerated point A the first oscillator wins and impose its frequency on the second one (as it was for $\gamma=0$ ). Above point A the latter dominates and in its turn impose its own frequency on the former. For bigger $\gamma$ only the latter regime remains.

Concerning the shape of the region, where this regime takes place ( $D_{2}^{1}$ in Fig. 14), our results show that it has two asymptotes: $\beta=1$ and $\beta=1+\gamma$, consequently the width of this region is simply $\gamma$.

To summarize the evolution of the regions, where different synchronization regimes take place, we present synchronization regions for different values of $\gamma$ in Fig. 17. As before, the region, where at least one synchronization regime exists, is marked by S (Fig. 17(a), (c) and (e)) and either has two separate parts (Fig. 17(a)) (if $0<\gamma \leq 2 \alpha$ ) or forms one continuous area (Fig. 17(e)) (if $\gamma>2 \alpha$ ), which has a branch between beating regime (B) and "oscillator death" (OD) regions. A comparison clearly shows that an increase of the amplitude mismatch $\gamma$ makes the synchronization region expand greatly. The width of it for fixed $\Delta$ is close to $\gamma$ for $\Delta \rightarrow \infty$. At the same time, the region of bistability $S^{2}$ is gradually substituted by the region, where the only stable rest state is the anti-phase one ${ }^{\pi} S^{1}$ (Fig. 17(b) and (d)). This eventually merges with the region of the in-phase synchronization regime ${ }^{0} S^{1}$ and forms the region $S^{1}$, where the only stable rest state vary its phase difference characteristic from in- to anti-phase continuously (Fig. 17(f)).

Transition to the beating regime. As before, we investigate changes in the averaged beating frequency $\omega_{\mathrm{b}}$ that follow the loss of synchronization. Comparing the results presented in Fig. 18(a) with those obtained in case $\gamma=0$, we observe two qualitatively new features: For fixed $\alpha=0$ (Fig. 18(a)) the smooth and the sharp transitions to phase drift alternate as $\beta$ is increased, i.e., an Andronov-Hopf bifurcation substitutes a saddle-node bifurcation for a while. The other notable feature of the case of non-zero amplitude mismatch is revealed on the neighbor diagram Fig. 18(b). When the coupling coefficients are no longer zero, increasing of one of them may lead to a synchronization break, even if every other parameter, including frequency mismatch, is fixed. This result is the consequence of the non-monotonous dependence of the synchronization threshold upon the coupling strength, which was already discussed in this section. 


\section{Conclusions}

In the present work we have performed analytical treatment and numerical simulations of Eqs. (1)-(5) to find and elucidate the effects that take place in the system of two non-identical van der Pol oscillators with diffusive non-scalar coupling, which combines conservative with dissipative terms.

We have studied two types of parameter mismatch and have found the following main results:

(i) Non-zero frequency mismatch, zero amplitude mismatch:

- We have found the existence of the regions of various bi-and multistability of synchronization and beating regimes in case when the oscillators differ only in the frequencies. According to our results, these complex regimes appear due to Andronov-Hopf bifurcations that govern the system.

- The effect of oscillator death remains existing in case of non-scalar coupling.

- Studying phase difference, frequency and amplitude characteristics of synchronization, we have found them to change substantially. Almost in the whole region, where each synchronization regime exists, its frequency remains approximately constant. Thus we are able to mark out three frequency values associated with the in- $\left(\omega_{\mathrm{s}}=\Delta / 2\right)$ and anti-phase $\left(\omega_{\mathrm{s}}=\Delta / 2+2 \alpha\right)$ regimes and synchronization near frequency of the first oscillator $\left(\omega_{\mathrm{s}}=\alpha\right)$. The latter regime has not been observed before and occurs due to the frequency asymmetry of the system, which appears when a conservative coupling is introduced $(\alpha \neq 0)$. This unusual regime may also be regarded as the analog of the frequency pull-in effect in the case of synchronization by the external signal.

- Studying the beating frequency, we have observed two qualitatively different types of synchronization break-up: "soft" and "hard". For increasing $\Delta$ from zero (synchronization regime) to its limit value, the beating frequency demonstrate either discontinuity or a smooth transition. The former is associated with a transformation of a limit cycle with bounded changes of the phase difference to one with its unbounded growth. Besides our results show that the larger are the coupling parameters, the sharper is the continuous transition to the beating regime.

- In general, the region where synchronization takes place enlarges monotonously as the conservative coupling is strengthened.

(ii) Non-zero frequency and amplitude mismatches:

- A small amplitude mismatch $\gamma$ leads to the appearance of a region of monostability of the anti-phase synchronization.

- The bi- and multistability regions decrease and disappear on behalf of the monostability region.

- A peculiar effect appears that for a certain range of $\gamma$ and $\Delta$ the increase of $\alpha$ or $\beta$ makes the unique synchronization regime cease existing for a while, in contrast to the fact that it has always led to the reverse effect of gaining synchronization before.

- We have also observed a continuous transition from the in- to the anti-phase regime (follows the increase of $\beta$ ) that takes place for quite big $\gamma$. The frequency of synchronization changes substantially inside the synchronization regime region, not only near the bifurcation, in contrast to the case of $\gamma=0$. The bigger is $\gamma$, the more distinct this tendency is.

- We have found an unusual effect that under certain conditions the oscillators become qualitatively unequal in course of interaction and the second one dominates the first one, all the while being only slightly "stronger" in the amplitude value, when isolated. Then, in spite of coupling coefficients and amplitude mismatch being fixed, synchronization persists in this system as the frequency mismatch is gradually increased at the expense of the "weaker" oscillator, its amplitude response being almost totally suppressed and the frequency of synchronization approaches the value the isolated second oscillator demonstrates. What is impressive 
about this effect, comparing to the one that takes place for zero $\gamma$, is the fact that it exist for every $\Delta$, whatever large it is, not only for fixed $\beta=1$, but for a range of the values of the latter, whose width has been shown to be $\gamma$. The values of coupling parameters that belong to the named interval should also be considered as optimal ones with respect to synchronization between non-identical systems.

- The beating frequency characteristics show two qualitatively new features comparing to the case of zero $\gamma$. The first is the non-monotonous dependence of the synchronization threshold upon the coupling strength. The second is the alteration between continuous and discontinuous dependencies of the averaged beating frequency upon the frequency mismatch value, as the coupling parameter $\beta$ is monotonously changed.

- Generally, the region of synchronization enlarges as the amplitude mismatch increases. The shape of this region acquires a new feature, specifically, there appears a branch between the beating regime and the oscillator death regions, which broadens following the increase of the amplitude mismatch (the width of it was shown to be $\gamma$ ).

The results we have obtained in course of our studies seem to be most interesting and reveal qualitatively new and unexpected properties of the system of coupled limit-cycle oscillators. As they are present in a classical system, we expect them to be characteristic of a wide class of interacting systems and they should be found in various experiments, too. The extension of these results to chains and lattices of coupled limit-cycle oscillators is a subject of future studies.

\section{Acknowledgements}

This work was supported by RFBR (Project Nos. 02-02-17573 and 03-02-17543) and INTAS (Project No. 01-867), MI also acknowledges financial support of RFBR (Project No. 03-02-06371) and "Dynasty" Foundation, GO also acknowledges financial support as Visiting Professor in Cognitive Science at the Potsdam University and IQN (DAAD).

\section{References}

[1] C. Hugenii, Horoloquim Oscilatorium, Paris, France, 1673.

[2] A.S. Pikovsky, M.G. Rosenblum, J. Kurths, Synchronization-A Universal Concept in Nonlinear Sciences, Cambridge University Press, Cambridge, 2001.

[3] S. Boccaletti, J. Kurths, G. Osipov, D.L. Valladares, C.S. Zhou, Synchronization of chaotic systems, Phys. Rep. 366 (2002) 1.

[4] E.V. Appleton, The automatic synchronization of triode oscillator, Proc. Camb. Philos. Soc. (Math. Phys. Sci.) 21 (1922) 231.

[5] B. van der Pol, Forced oscillators in a circuit with nonlinear resistance (reception with reactive triode), Philos. Mag. 3 (1927) 64.

[6] A.A. Andronov, A.A. Vitt, On mathematical theory of entrainment, Z. Prikl. Fiz. (J. Appl. Phys.) 7 (1930) 3 (in Russian).

[7] A.A. Andronov, A.A. Witt, Zur Theorie des Mitnehmens van der Pol, Arch. Elektrotechn. 24 (1930) 99.

[8] L.I. Mandelshtam, N.D. Papaleksi, On the $n$th kind resonance phenomena, in: Collected Works by L.I. Mandelshtam, vol. 2, Izd. Akademii Nauk, Moscow, 1947, p. 13 (in Russian).

[9] A. Mayer, On the theory of coupled vibrations of two self-excited generators, Proc. Gorky State Univ. 2 (1935) 3 (in Russian).

[10] V.I. Gaponov, Two coupled generators with soft self-excitation, Zh. Tech. Fiz. (J. Tech. Phys.) 6 (1936) 5 (in Russian).

[11] M.L. Cartwright, J.E. Littlewood, On nonlinear differential equations of second order, J. London Math. Soc. 20 (1945) 180.

[12] M.L. Cartwright, Forced oscillations in nearly sinusoidal systems, J. Inst. Electr. Eng. 95 (1948) 88.

[13] A.S. Bremsen, I.S. Feinberg, Analysis of functioning of two coupled relaxation generators, Zh. Tech. Fiz. (J. Tech. Phys.) 11 (1941) 959 (in Russian).

[14] K.F. Teodorchik, On the theory of synchronization of relaxation self-oscillations, Dokl. Akad. Nauk SSSR (Sov. Phys. Dokl.) 40 (1943) 63 (in Russian).

[15] D.W. Storti, R.H. Rand, Dynamics of two strongly coupled van der Pol oscillators, Int. J. Non-Linear Mech. 17 (3) (1982) 143.

[16] R.H. Rand, P.J. Holmes, Bifurcation of periodic motions in two weakly coupled van der Pol oscillators, Int. J. Non-Linear Mech. 15 (1980) 387. 
[17] G.B. Ermentrout, Oscillation death in populations of "all to all" coupled nonlinear oscillators, Physica D 41 (1990) 219.

[18] D.G. Aronson, G.B. Ermentrout, N. Kopell, Amplitude response of coupled oscillators, Physica D 41 (1990) 403.

[19] M. Poliashenko, S.R. McKay, C.W. Smith, Chaos and nonisochronism in weakly coupled nonlinear oscillators, Phys. Rev. A 44 (6) (1991) 3452.

[20] G.V. Osipov, M.M. Sushchik, Synchronized clusters and multistability in arrays of oscillators with different natural frequencies, Phys. Rev. E 58 (1998) 7198.

[21] T.E. Vadivasova, G.I. Strelkova, V.S. Anishchenko, Phase-frequency synchronization in a chain of periodic oscillators in the presence of noise and harmonic forcing, Phys. Rev. E 63 (2001) 036225.

[22] S.E. de Sousa Pinto, S.R. Lopes, R.L. Viana, Collective behavior in a chain of van der Pol oscillators with power-low coupling, Physica A 303 (2002) 339.

[23] H. Sakaguchi, Desynchronization in a self-oscillating medium, Prog. Theoret. Phys. 80 (5) (1988) 743.

[24] T. Chacraborty, R.H. Rand, The transition from phase locking to drift in a system of two weakly coupled van der Pol oscillators, Int. J. Non-Linear Mech. 23 (1988) 369. 